\title{
Resummation of the hadronic tau decay width with the modified Borel transform method
}

\author{
Gorazd Cvetič ${ }^{1 *}$, Claudio Dib ${ }^{1 \dagger}$, Taekoon Lee ${ }^{2 \ddagger}$, and Iván Schmidt ${ }^{1 \S}$ \\ ${ }^{1}$ Department of Physics, Universidad Técnica Federico Santa María, Valparaíso, Chile \\ ${ }^{2}$ Department of Physics, KAIST, Daejon 305-701, Korea
}

(October 26, 2018)

\begin{abstract}
A modified Borel transform of the Adler function is used to resum the hadronic tau decay width ratio. In contrast with the ordinary Borel transform, the integrand of the Borel integral is renormalization-scale invariant. We use an ansatz which explicitly accounts for the structure of the leading infrared renormalon. Further, we use judiciously chosen conformal transformations for the Borel variable, in order to map sufficiently away from the origin the other ultraviolet and infrared renormalon singularities. In addition, we apply Padé approximants for the corresponding truncated perturbation series of the modified Borel transform, in order to further accelerate the convergence. Comparing the results with the presently available experimental data on the tau hadronic decay width ratio, we obtain $\alpha_{\mathrm{s}}\left(M_{\mathrm{z}}^{2}\right)=0.1192 \pm 0.0007_{\text {exp. }} \pm 0.0010_{\mathrm{EW}+\mathrm{CKM}} \pm 0.0009_{\text {th. }} \pm 0.0003_{\text {evol. }}$. These predictions virtually agree with those of our previous resummations where we used ordinary Borel transforms instead.
\end{abstract}

PACS number(s): 13.35.Dx, 11.15.-q, 11.15.Tk

*cvetic@fis.utfsm.cl

${ }^{\dagger}$ cdib@fis.utfsm.cl

‡tlee@muon.kaist.ac.kr

§ischmidt@fis.utfsm.cl 


\section{INTRODUCTION}

Extensive perturbative calculations in QCD have made available the truncated perturbation series (TPS) of various observables to the next-to-next-to-leading order (NNLO), i.e., including the terms $\sim \alpha_{s}^{3}$. There is a longstanding problem of how to extract (resum), in a reasonable manner, the values of such observables $S\left(\alpha_{s}\right)$ as functions of the strong

coupling parameter $\alpha_{s}^{\overline{\mathrm{MS}}}\left(M_{\mathrm{z}}^{2}\right)$. Many of the resummation methods are based solely on the available TPS $S_{[3]}\left(\alpha_{s}\right)$. Some of these methods eliminate the (unphysical) renormalizationscale (RScl) and renormalization-scheme (RSch) dependence of the TPS by fixing the RScl and RSch in the TPS itself. Among them are: the RScl fixing of Brodsky, Lepage and Mackenzie (BLM), motivated by large $-n_{f}$ arguments [1]; the principle of minimal sensitivity (PMS) [2]; and effective charge method (ECH) [3 5]. Some of the more recent methods are: the approaches using "commensurate scale relations" [6]; ECH-related approaches [7 [10]; a method using an analytic form of the coupling parameter [11]; a method using expansions in the two-loop coupling parameter [12]; a method which disentangles the running coupling and conformal effects (skeleton coefficients) [13]; methods using conformal transformations either for the coupling parameter [14] or for the Borel expansion parameter [15,16]; the method of Padé approximants (PA's) [17 [19]; an RScl-invariant [20] and RScl- and RSch-invariant extension of the PA-approach [21].

Some of the aforementioned approaches allow for incorporation of additional, nonperturbative, information on the observable, e.g., the information on the location of the dominant infrared (IR) renormalon singularity [21] via the fixing of the remaining free parameter in the approximant, or the information on the location of various renormalon singularities via leading- $\beta_{0}$ resummation [8,9]. Full information on the known leading IR renormalon structure can be incorporated in a natural way through an explicit ansatz, in the approaches using Borel transformations of the considered or of the associated observable (see, e.g., [22]). Incorporation of the available leading renormalon information appears to influence significantly the numerical predictions, particularly for low-energy (low- $Q^{2}$ ) QCD observables, through a more accurate description of the Borel amplitude in the region between the origin and the leading IR renormalon singularity. This method has an advantage over the widely used (unresummed) Operator Product Expansion (OPE) approach [23], in which the perturbative contributions are usually taken as TPS, and additional nonperturbative terms $\propto 1 / Q^{2 n}$ appear (cf. [24] and references therein). Since the perturbation series is divergent, the latter approach has the problem that there is no natural separation between perturbative contributions and power-suppressed nonperturbative terms, a problem which does not occur in the former method. However, the Borel-resummed value for an observable, with the renormalon information incorporated in it (e.g., via an explicit ansatz), in principle still does not represent the full value of the observable, because power-suppressed terms should be added to it. Dispersive models ( 25] and references therein) contain and predict the OPE-type power-suppressed terms. Recently, it has been argued [26] that the power-suppressed terms can be obtained from the knowledge of the perturbation series, or parts thereof, and of the IR renormalon structure of the observable.

In all these resummation approaches, for obvious reasons, it is highly preferable to consider QCD observables whose values are presently known at a high precision level. Presently, high precision experimental data on $\tau$ lepton decay widths 27 32 are available. The non- 
strange hadronic $\tau$ decay width ratio $R_{\tau}(\triangle S=0)$ can be obtained by using constrained fit values [33] of the (basis modes) leptonic branching ratios $B_{e} \equiv B\left(\tau^{-} \rightarrow e^{-} \bar{\nu}_{e} \nu_{\tau}\right)=$ $(17.83 \pm 0.06) \times 10^{-2}$ and $B_{\mu} \equiv B\left(\tau^{-} \rightarrow \mu^{-} \bar{\nu}_{\mu} \nu_{\tau}\right)=(17.37 \pm 0.07) \times 10^{-2}$ of $\tau$, and subtracting the strangeness-changing part [32] $R_{\tau}(\triangle S \neq 0)=0.1630 \pm 0.0057$

$$
\begin{aligned}
R_{\tau}(\triangle S=0) & \equiv \frac{\Gamma\left(\tau^{-} \rightarrow \nu_{\tau} \text { hadrons }(\gamma)\right)}{\Gamma\left(\tau^{-} \rightarrow \nu_{\tau} e^{-} \bar{\nu}_{e}(\gamma)\right)}-R_{\tau}(\triangle S \neq 0) \\
& =\frac{\left(1-B_{e}-B_{\mu}\right)}{B_{e}}-R_{\tau}(\triangle S \neq 0)=3.4713 \pm 0.0171
\end{aligned}
$$

The above ratio is a QCD observable at relatively low momenta $\sqrt{q^{2}} \sim m_{\tau} \approx 1.8 \mathrm{GeV}$, experimentally known to high precision, thus presenting an experimental challenge to the theory. The challenge consists in predicting the strong coupling constant $\alpha_{s}^{\overline{\mathrm{MS}}}\left(M_{Z}^{2}\right)$ so that the theoretical uncertainty $\left(\delta \alpha_{s}\right)_{\text {th. }}$, which partly originates from the uncertainty of the method of resummation and partly from the uncertainty of the associated Adler function, is smaller or comparable to the uncertainty $\left(\delta \alpha_{s}\right)_{\text {exp. }}$ originating from the small experimental uncertainty $\delta R_{\tau} \approx \pm 0.017$ given above.

There is now a wealth of theoretical results [24,34 40] available on the observable (2), and, in particular, on the associated Adler function $D\left(Q^{2}\right)$ - perturbative as well as nonperturbative. The main QCD theoretical problem connected with the observable (2) is that the pertaining momenta are somewhat low $|Q| \sim m_{\tau}(\approx 1.8 \mathrm{GeV})$, and so the relevant coupling parameter $\alpha_{s}\left(Q^{2}\right)$ in the perturbation expansion is large. It is thus important to take into account, in any resummation procedure for $R_{\tau}$ and/or $D\left(Q^{2}\right)$, not just the known perturbative coefficients, but also a significant part of the nonperturbative information, i.e., the leading infrared renormalon. On the other hand, the nonperturbative contributions to $R_{\tau}$ that are represented by power-suppressed OPE terms (apart from the well known quark mass contributions) have been shown to be consistent with zero in the ALEPH analysis [27].

One version of this program was carried out in our previous work [22]. There we first used the known information on the leading infrared (IR) renormalon and on the corresponding 1/Q $Q^{2}$-suppressed term of the OPE for the Adler function $D\left(Q^{2}\right)$, in order to predict the $\mathcal{O}\left(\alpha_{s}^{4}\right)$ coefficient $d_{3}\left(Q^{2}\right)$ of $D\left(Q^{2}\right)$. Using this prediction, and judiciously chosen conformal transformations, we resummed $R_{\tau}$ by employing an ordinary Borel transform $\widetilde{D}(b)$ of the Adler function with an ansatz which explicitly accounted for the leading IR renormalon structure. Comparing the obtained expression with the experimental values (2), we obtained definite predictions for the strong coupling parameter $\alpha_{s}\left(M_{\mathrm{z}}^{2}\right)=0.1193 \pm 0.0007_{\text {exp. }} \pm 0.0010_{\mathrm{EW}+\mathrm{CKM}} \pm 0.0009_{\text {meth. }} \pm 0.0003_{\text {evol. }}$. We fixed the RScl by the principle of minimal sensitivity (PMS) applied to the approximant, and by choosing the $\overline{\mathrm{MS}} \mathrm{RSch}$.

In the present work instead, in order to obtain a cross-check of the predictions, we employ not ordinary Borel transforms but modified Borel transforms of $D\left(Q^{2}\right)$. Such Borel transforms were introduced by Grunberg [41], on the basis of the modified Borel transforms of Ref. [42]. One attractive feature of Grunberg's transforms is that the integrand in the Borel integral is RScl-independent, in contrast to the case of the ordinary Borel transforms. Another interesting feature is that they represent integral transformations of a significantly different form than the ordinary Borel transformation, and have therefore a different singularity structure. Therefore, their application to the hadronic tau decay width ratio and the 
subsequent extraction of the prediction for $\alpha_{s}\left(M_{\mathrm{z}}^{2}\right)$ could represent a powerful cross-check of the results of previous work 22] based on ordinary Borel transformations.

In Sec. II we recall the known basic theoretical formulas for $R_{\tau}(\triangle S=0)$ and the associated Adler functions, as well as the reduction to the massless QCD observable $r_{\tau}$. In Sec. III, we present the modified Borel transforms $\bar{D}(b)$ of the massless Adler function $D\left(Q^{2}\right)$. We refer to the Appendix for details about $\bar{D}(b)$. In Sec. IV we perform the resummation for $r_{\tau}$, by contour integration of $D\left(Q^{2}\right)$ in the complex momentum plane, explicitly accounting for the leading IR renormalon structure of $\bar{D}(b)$ through an ansatz, choosing conformal transformations of the Borel variable $b$ to map away other singularities of $\bar{D}(b)$, and employing Padé approximants. In Sec. $\nabla$ we compare the obtained expressions with the experimental results and determine $\alpha_{s}\left(m_{\tau}^{2}\right)$ and $\alpha_{s}\left(M_{\mathrm{z}}^{2}\right)$. We further estimate the theoretical uncertainties of the prediction. Sec. VI contains a summary and brief discussion of the differences between our results and those of other analyses of $R_{\tau}$.

\section{THE KNOWN BASIC FORMULAS, REDUCTION TO MASSLESS QCD}

The restriction $\triangle S=0$ in Eq. (2) means that only hadrons with quarks $u$ and $d$ are produced. Thus the observable (2) is already close to being massless. This fact removes some of the complications in the theoretical analysis.

The ratio (2) can be expressed, via the application of a variant of the optical theorem, and the subsequent use of Cauchy's theorem and integration by parts, as a contour integral in the complex momentum plane (see, for example, Refs. [24,40]):

$$
\begin{aligned}
& r_{\tau}(\triangle S=0) \equiv \frac{R_{\tau}(\triangle S=0)}{3\left|V_{u d}\right|^{2}\left(1+\delta_{\mathrm{EW}}\right)}-\left(1+\delta_{\mathrm{EW}}^{\prime}\right) \\
& =(-\pi \mathrm{i}) \int_{|s|=m_{\tau}^{2}} \frac{d s}{s}\left(1-\frac{s}{m_{\tau}^{2}}\right)^{3}\left[\left(1+\frac{s}{m_{\tau}^{2}}\right) D^{\mathrm{L}+\mathrm{T}}(-s)+\frac{4}{3} D^{\mathrm{L}}(-s)\right]-1 .
\end{aligned}
$$

Here we factored out, for convenience, the square of the Cabibbo-Kobayashi-Maskawa (CKM) matrix element $\left|V_{u d}\right|$, the electroweak $(\mathrm{EW})$ correction parameter $\delta_{\mathrm{EW}}=0.0194 \pm$ 0.0050 43,44,24, and the residual EW correction parameter $\delta_{\mathrm{EW}}^{\prime}=0.0010$ [43]. The contour integration in Eq. (1) is counterclockwise in the complex $s$-plane, and the general Adler functions $D^{\mathrm{L}+\mathrm{T}}$ and $D^{\mathrm{L}}$ are related to the $\mathrm{V}+\mathrm{A}$ current-current correlation functions as:

$$
\begin{aligned}
D^{\mathrm{L}+\mathrm{T}}(-s) & =-s \frac{d}{d s} \sum_{J=0,1}\left(\Pi_{u d, V}^{(J)}(s)+\Pi_{u d, A}^{(J)}(s)\right) \\
D^{\mathrm{L}}(-s) & =\frac{s}{m_{\tau}^{2}} \frac{d}{d s}\left[s\left(\Pi_{u d, V}^{(0)}(s)+\Pi_{u d, A}^{(0)}(s)\right)\right] .
\end{aligned}
$$

Here, $J$ is the spin of the hadronic system in its rest frame $(\mathrm{L}: J=0 ; \mathrm{T}: J=1) ; \Pi_{u d, V / A}^{(J)}$ are the components in the Lorentz decomposition

$$
\Pi_{u d, V / A}^{\mu \nu}(q)=\left(-g^{\mu \nu} q^{2}+q^{\mu} q^{\nu}\right) \Pi_{u d, V / A}^{(1)}\left(q^{2}\right)+q^{\mu} q^{\nu} \Pi_{u d, V / A}^{(0)}\left(q^{2}\right)
$$

of the two-point correlation functions $\Pi_{u d, V / A}^{\mu \nu}$ of the vector $V_{u d}^{\mu}=\bar{d} \gamma^{\mu} u$ and axial-vector $A_{u d}^{\mu}=\bar{d} \gamma^{\mu} \gamma_{5} u$ (color-singlet) currents 


$$
\begin{aligned}
-\mathrm{i} \Pi_{u d, V}^{\mu \nu}(q) & =\int d^{4} x e^{\mathrm{i} q \cdot x}\left\langle 0\left|\mathrm{~T}\left\{V_{u d}^{\mu}(x) V_{u d}^{\nu}(0)^{\dagger}\right\}\right| 0\right\rangle, \\
-\mathrm{i} \Pi_{u d, A}^{\mu \nu}(q) & =\int d^{4} x e^{\mathrm{i} q \cdot x}\left\langle 0\left|\mathrm{~T}\left\{A_{u d}^{\mu}(x) A_{u d}^{\nu}(0)^{\dagger}\right\}\right| 0\right\rangle,
\end{aligned}
$$

In the massless quark limit $\left(m_{u, d} \rightarrow 0\right), D^{\mathrm{L}}(s)$ vanishes and the vector and axial-vector contributions to $D^{\mathrm{L}+\mathrm{T}}$ become equal in perturbation and $D^{\mathrm{L}+\mathrm{T}}(-s) \rightarrow(1+D(-s)) /\left(2 \pi^{2}\right)$, where $D\left(Q^{2}\right)$ is the canonically normalized massless Adler function with the perturbative expansion!

$$
D\left(Q^{2}\right)=a\left[1+\sum_{n=1}^{\infty} d_{n} a^{n}\right] .
$$

Here, $a=\alpha_{s}\left(\mu^{2} ; c_{2}, c_{3}, \ldots\right) / \pi$ is the QCD couplant at the renormalization scale (RScl) $\mu^{2}$ and in the renormalization scheme (RSch) characterized by the coefficients $c_{j}(j \geq 2)$ in the beta function

$$
\mu^{2} \frac{d}{d \mu^{2}} a \equiv \beta(a)=-\beta_{0} a^{2}\left[1+c_{1} a+c_{2} a^{2}+\cdots\right] .
$$

Here, $\beta_{0}=\left(11-2 n_{f} / 3\right) / 4$ and $c_{1}=\left(102-38 n_{f} / 3\right) /\left(16 \beta_{0}\right)$ are two universal constants which depend only on the number of active quark flavors $n_{f}$. The Adler functions are quasiobservables, in the sense that they are independent of the RScl and RSch.

In order to apply the massless QCD analysis to $r_{\tau}(\triangle S=0)$ (4), we have to subtract from it the quark mass $\left(m_{u, d} \neq 0\right)$ contributions. This can be carried out [24] within an operator product expansion (OPE). The largest quark mass contributions are quark condensate terms of dimension $d=4\left(\propto 1 / m_{\tau}^{4}\right)$

$$
\begin{aligned}
& \delta r_{\tau}(\triangle S=0)_{m_{u, d} \neq 0} \approx 16 \pi^{2} \frac{\left(m_{u}+m_{d}\right)\langle\bar{q} q\rangle}{m_{\tau}^{4}}\left[1+\frac{23}{8}\left(\frac{\alpha_{s}\left(m_{\tau}^{2}\right)}{\pi}\right)^{2}\right] \\
& \approx-\frac{16 \pi^{2} f_{\pi}^{2} m_{\pi}^{2}}{m_{\tau}^{4}}\left[1+\frac{23}{8}\left(\frac{\alpha_{s}\left(m_{\tau}^{2}\right)}{\pi}\right)^{2}\right] \approx-0.00265 \times(1+0.03) \approx-0.0027 .
\end{aligned}
$$

In Eq. (12) we denoted $\langle\bar{q} q\rangle \equiv\langle\bar{u} u\rangle \approx\langle\bar{d} d\rangle$. Here renormalization scale can be taken to be $\mu \approx m_{\tau}$. In Eq. (13) we used the PCAC relation $\left(m_{u}+m_{d}\right)\langle\bar{q} q\rangle \approx-f_{\pi}^{2} m_{\pi}^{2}\left(f_{\pi}=92.4 \pm 0.3\right.$ $\left.\mathrm{MeV} ; m_{\pi^{-}}=139.6 \mathrm{MeV}\right)$. There are corrections to this relation and to expression (13) of the order $\sim m_{u, d}^{2} / m_{\tau}^{2}$, i.e., of the order of the OPE $d=2$ terms which can reach, at most, values $\sim 10^{-4}$. In obtaining the numerical value in Eq. (13), we further used $m_{\tau}=1777 \mathrm{MeV}$ and $\alpha_{s}\left(m_{\tau}^{2}, \overline{\mathrm{MS}}\right) \approx 0.32$.

The OPE approach of 24] includes other power-suppressed nonperturbative terms that contribute to $r_{\tau}$ but do not stem from quark masses: the $d=4$ gluon condensate term, and the $d=6$ term. The latter term could be large, but it has also comparably large uncertainties

1 The $(u d)$ Adler functions $D^{\mathrm{L}+\mathrm{T}}$ and $D^{\mathrm{L}}$ (5)-(6) usually include, by convention, the additional CKM factor $\left|V_{u d}\right|^{2}$ (e.g., see Refs. [40]). 
[24]. The gluon condensate contribution to $r_{\tau}$ in the OPE is $\alpha_{s}^{2}$-suppressed. The ALEPH analysis [27] indicates that these $d=4,6$ nonperturbative contributions to $r_{\tau}$ are consistent with the value zero, $\delta r_{\tau}\left(\mathrm{NP} ; m_{u, d}=0\right)=0.000 \pm 0.004$. We should keep in mind, however, that the ALEPH analysis assumed that the part of the associated Adler function which has no power-suppressed terms is a $\left(\mathrm{N}^{3} \mathrm{LO}\right)$ TPS, while we will perform resummations of this part by taking into account its renormalon singularity structure. Nonetheless, we consider the ALEPH analysis as indicative that even in our resummation framework the powersuppressed OPE-type terms in $r_{\tau}$ (apart from the quark mass terms) are either consistent with zero or very small. Therefore, we will ignore in our analysis of $r_{\tau}$ any OPE powersuppressed nonperturbative terms other than those in Eqs. (12)-(13).

We thus regard, in our framework, as nonperturbative massless contributions only those contributions which appear as a consequence of explicit IR renormalon structure of the Borel transforms in the resummation. For example, the leading IR renormalon of the Adler function $D\left(Q^{2}\right)$ (which we will account for) gives contributions to $D\left(Q^{2}\right)$ which can be partially represented as a $d=4$ power-suppressed term $\propto 1 / Q^{4}$. This, however, does not necessarily mean that there is no additional, genuine OPE-type $d=4$ term $\left(\propto\langle a G G\rangle / Q^{4}\right)$ in $D\left(Q^{2}\right)$. The uncertainties of the OPE $d=4,6$ massless terms as given by ALEPH are large enough to accommodate the possibility of significant nonzero values of these terms. For example, $\langle a G G\rangle=0.001 \pm 0.015 \mathrm{GeV}^{4}$. Toward the end of Sec. $\nabla$ we will briefly discuss how the latter uncertainties would influence the final prediction for $\alpha_{s}$ in our analysis.

The ALEPH analysis further assumed that massless $d=2$ terms $\left(\propto 1 / Q^{2}\right)$ are not present in the Adler function (and thus in $r_{\tau}$ ), as suggested by the OPE. Such terms were suggested by the authors of Refs. [45,46] as an effective tachyonic gluon mass contribution reflecting nonperturbative short-distance QCD. However, the authors of Ref. [47] showed that the strength of such terms is consistent with zero.

When subtracting the quark mass contributions (12)-(13) from Eq. (44), we end up with the massless QCD observable

$$
\begin{aligned}
r_{\tau} & \equiv r_{\tau}\left(\triangle S=0 ; m_{u, d}=0\right)=r_{\tau}(\triangle S=0)-\delta r_{\tau}(\triangle S=0)_{m_{u, d} \neq 0} \\
& =-\frac{\mathrm{i}}{2 \pi} \int_{|s|=m_{\tau}^{2}} \frac{d s}{s}\left(1-\frac{s}{m_{\tau}^{2}}\right)^{3}\left(1+\frac{s}{m_{\tau}^{2}}\right) D(-s), \\
& =\frac{1}{2 \pi} \int_{-\pi}^{\pi} d y\left(1+e^{\mathrm{i} y}\right)^{3}\left(1-e^{\mathrm{i} y}\right) D\left(-s=m_{\tau}^{2} e^{\mathrm{i} y}\right),
\end{aligned}
$$

with the canonically normalized massless Adler function $D\left(Q^{2} \equiv-s\right)$ defined in Eq. (10).

The perturbation coefficients $d_{j}=d_{j}\left(\mu^{2} / Q^{2} ; c_{2}, \ldots, c_{j}\right)$ in Eq. (10) depend on the RScl $\mu^{2}$ and the RSch parameters $c_{j}$ in a known specific manner, because $D\left(Q^{2}\right)$ is RScl- and RSch-independent. Knowing them at a specific RScl and RSch, we know them at any RScl and RSch - see Eqs. (A.5)-(A.8) in the Appendix. The first two coefficients have already been calculated, and in the $\overline{\mathrm{MS}} \mathrm{RSch}$ and at $\mathrm{RScl} \mu^{2}=Q^{2}$, for $n_{f}=3$, they are: $d_{1}^{(0)}=1.6398$

2 They did this by fitting a $d=2$ finite energy sum rule, which is apparently well satisfied at the used relevant scales 48,49], to the new ALEPH data on spectral functions extracted from the $\tau$ decay measurements. 
[50]; $d_{2}^{(0)}=6.3710$ [51] (the superscript (0) denotes the value at the aforementioned RScl and RSch).

In order to extract the experimental value of $r_{\tau}$ (14), according to Eq. (3) we need to use the values $R_{\tau}(\triangle S=0)$ of Eq. (2),$\delta_{\mathrm{EW}}=0.0194 \pm 0.0050$ [43, 144,24], $\delta_{\mathrm{EW}}^{\prime}=0.0010$ [43], $\delta r_{\tau}(\triangle S \neq 0)_{m_{u, d} \neq 0}(13)$, and in addition the value of the CKM matrix element $\left|V_{u d}\right|$, which we take as

$$
\left|V_{u d}\right|=0.9749 \pm 0.0021
$$

This leads us, via Eqs. (21), (3) and (13), to the following values for the massless QCD observable (14)

$$
\begin{aligned}
r_{\tau} & \equiv r_{\tau}\left(\triangle S=0 ; m_{u, d}=0\right) \\
& =0.1960 \pm 0.0059_{\text {exp. }} \pm 0.0059_{\mathrm{EW}} \pm 0.0051_{\mathrm{CKM}} \\
& =0.1960 \pm 0.0098 .
\end{aligned}
$$

In Eq. (19), the three uncertainties of Eq. (18) were added in quadrature.

The values (18), together with the contour integral expression (16), will be the starting point for our massless QCD resummation analyses of hadronic $\tau$ decays.

\section{MODIFIED BOREL TRANSFORMS}

The most straightforward way to perform the resummation for $r_{\tau}$ given in Eqs. (15)(16) would be to insert the known truncated perturbation series (TPS) for $D(-s)$ given in Eq. (10) and perform the momentum-contour integration, i.e., the approach of [39]. The method is, firstly, fraught with ambiguities from the choice of RScl and RSch. The final result contains residual, but significant, RScl and RSch dependence, due to the truncation of the series in Eq. (10). Secondly, the method does not incorporate the known renormalon structure of the Adler function's Borel transform $\widetilde{D}(b)$.

In a previous paper [22], two of us addressed the second problem, by employing in the resummation a (ordinary) Borel transform $\widetilde{D}(b)$ that includes the leading IR renormalon via the ansatz $\widetilde{D}(b)=R(b) /(1-b / 2)^{1+\nu}$, and by introducing in addition conformal transformations $b=b(w)$ in order to map sufficiently far away from the origin the singularities of the UV (and the remaining IR) renormalons. However, the integrand in the (ordinary) Borel integral is RScl- and RSch-dependent. This can be inferred from the definition

\footnotetext{
${ }^{3}$ The standard model (SM) unitarity-constrained fit predicts $\left|V_{u d}\right|=0.9749 \pm 0.0008$ [33]. However, the values extracted from the decays of mirror nuclei are lower: $\left|V_{u d}\right|=0.9740 \pm 0.0010$. This extraction has significant theoretical uncertainties (see [33] for further References). The values extracted from neutron decays are even lower $\left|V_{u d}\right|=0.9728 \pm 0.0012$ ( [33] and References therein), but appear to have smaller theoretical uncertainties. In view of all these considerations, we take in our analysis the value range as given in Eq. (17). Here, the central value is from the unitarityconstrained fit, and the uncertainty is increased so that (17) covers all the values from the decays of mirror nuclei and the upper half of the interval from neutron decays.
} 


$$
D\left(Q^{2}\right)=\frac{1}{\beta_{0}} \int_{0}^{\infty} d b \exp \left[-\frac{b}{\beta_{0} a\left(\mu^{2} ; c_{2}, \ldots\right)}\right] \widetilde{D}\left(b ; \mu^{2} / Q^{2}, c_{2}, \ldots\right)
$$

and from the expansion of the transform around the origin

$$
\widetilde{D}\left(b ; \mu^{2} / Q^{2}, c_{2}, \ldots\right)=1+\frac{d_{1}\left(\mu^{2} / Q^{2}\right)}{1 !}\left(\frac{b}{\beta_{0}}\right)+\sum_{n=2}^{\infty} \frac{d_{n}\left(\mu^{2} / Q^{2} ; c_{2}, \ldots, c_{n}\right)}{n !}\left(\frac{b}{\beta_{0}}\right)^{n} .
$$

For example, direct application of the derivative with respect to the $\mathrm{RScl} \mu^{2}$ gives

$$
\begin{aligned}
\frac{\partial}{\beta_{0} \partial \ln \mu^{2}}\left[\mathrm{e}^{-b /\left(\beta_{0} a\right)} \widetilde{D}(b)\right]= & \mathrm{e}^{-b /\left(\beta_{0} a\right)}\left\{\left[\frac{\beta(a)}{\beta_{0} a^{2}}+1\right] \frac{b}{\beta_{0}}+\left[d_{1}\left(\frac{\beta(a)}{\beta_{0} a^{2}}+1\right)+\frac{c_{1}}{2}\right] \frac{1}{1 !}\left(\frac{b}{\beta_{0}}\right)^{2}\right. \\
& \left.+\left[d_{2}\left(\frac{\beta(a)}{\beta_{0} a^{2}}+1\right)+\frac{1}{3}\left(2 c_{1} d_{1}+c_{2}\right)\right] \frac{1}{2 !}\left(\frac{b}{\beta_{0}}\right)^{3}+\cdots\right\},
\end{aligned}
$$

using the notations of Eq. (11). Once going beyond the one-loop approximation of the RGE-evolution (11), the integrand is RScl-dependent. If we knew the exact expression of the integrand, the total integral (20) would be RScl-independent. However, since we have a TPS available for $\widetilde{D}(b)$, we are forced to use a TPS for $R(b) \equiv(1-b / 2)^{1+\nu} \widetilde{D}(b)$. This truncation then results in the residual RScl and RSch dependence of the resummed result for $D\left(Q^{2}\right)$ and thus for $r_{\tau}$. In [22] we fixed the RScl-parameter $\xi^{2} \equiv \mu^{2} / Q^{2}$ according to the principle of minimal sensitivity (PMS), i.e., $\partial r_{\tau} / \partial \xi^{2}=0$. The RSch was chosen to be $\overline{\mathrm{MS}}$.

In the present paper we apply, instead of the ordinary Borel transform, several variants of the modified Borel transform $\bar{D}(b)$ of the Adler function $D\left(Q^{2}\right)$. The integrand will be RSclindependent. This Borel transform was introduced for QCD and QED (quasi)observables by Grunberg [41], who in turn constructed them on the basis of the modified Borel transformations of Ref. [42]. The integral transformation for $\bar{D}(b)$ is written in the form

$$
D\left(Q^{2}\right)=\frac{1}{\beta_{0}} \int_{0}^{\infty} d b \exp \left[-\frac{\left(\rho_{1}\left(Q^{2}\right)+\tilde{c}\right) b}{\beta_{0}}\right] \bar{D}(b ; \tilde{c}) .
$$

Here, $\bar{D}(b ; \tilde{c})=\exp \left(\tilde{c} b / \beta_{0}\right) \bar{D}(b ; 0)$ and has no $Q^{2}$-dependence; $\tilde{c}$ is a specific arbitrary constant; $\rho_{1}$ is the first RScl and RSch invariant [2] of the Adler function

$$
\rho_{1}\left(Q^{2}\right)=-d_{1}\left(\mu^{2} / Q^{2}\right)+\beta_{0} \ln \frac{\mu^{2}}{\widetilde{\Lambda}^{2}}=\beta_{0} \ln \frac{Q^{2}}{\bar{\Lambda}^{2}}
$$

where $\widetilde{\Lambda}$ is the universal scale appearing in the Stevenson's equation $\|2\|$, and $\bar{\Lambda}$ is a scale which depends on the observable but is RScl- and RSch-independent. We note that $\rho_{1}\left(Q^{2}\right)=$ $\left[1 / a^{(1-\text { loop })}\left(Q^{2}\right)+c\right]$, where $c$ is a constant. Therefore, $\bar{D}(b ; \tilde{c})$ reduces to the ordinary Borel transform $\widetilde{D}\left(b ; \mu^{2} / Q^{2}=1, \ldots\right)$ times the factor $\exp \left[(c+\tilde{c}) b / \beta_{0}\right]$, when higher than one-loop effects are ignored (large- $\beta_{0}$ approximation).

Since $D\left(Q^{2}\right)$ and $\rho_{1}\left(Q^{2}\right)$ in Eq. (23) are RScl- and RSch-independent, so $\bar{D}(b ; \tilde{c})$ possesses no explicit RScl- and RSch-dependence. However, as shown in the Appendix, the modified Borel transformation (23) is one in a large class of Borel transformations, where each of them corresponds to a specific choice of the RScl and RSch. The transformation (23) is obtained from this class by choosing the so called modified 't Hooft RSch (mtH RSch: $c_{j}=c_{1}^{j}$, 
$j=2,3, \ldots)$. We also show that in this case the constant $\tilde{c}$ is in fact the RScl parameter $\tilde{c}=$ $\beta_{0} \ln \left(\xi^{2}\right)$, where $\xi^{2} \equiv \mu^{2} / Q^{2}$. This special Borel transformation has the remarkable property that the integrand in the Borel integral is RScl-independent ( $\tilde{c}$-independent). Therefore, we can call it RScl-independent Borel transformation, since the change of the RScl only changes the convention of separating the (RScl-invariant) integrand in Eq. (23) into two factors.

Yet another useful property of the modified Borel transform (23) is the following: due to the very simple $Q^{2}$-dependence of the integrand in Eq. (23), the contour integration (16) in the complex momentum plane can be performed analytically, leading to a rather simple expression for the observable $r_{\tau}$, as will be shown in the next section. This is not possible if the mtH RSch is abandoned.

The coefficients of expansion of $\bar{D}(b ; \tilde{c})$ in Eq. (23) around $b=0$ are related with those of the expansion of $D\left(Q^{2}\right)$ most easily when we use for the latter expansion the specific $\mathrm{mtH}$ $\operatorname{RSch} c_{k}=c_{1}^{k}(k \geq 2)$ and RScl $\mu^{2}=Q^{2}$

$$
\begin{aligned}
\bar{D}(b ; \tilde{c})= & \frac{\left(c_{1} b / \beta_{0}\right)^{c_{1} b / \beta_{0}}}{\Gamma\left(1+c_{1} b / \beta_{0}\right)} \exp \left(\frac{\left(\tilde{c}-\tilde{d}_{1}\right) b}{\beta_{0}}\right)\left\{1+\frac{\left(\tilde{d}_{1}-c_{1}\right)}{\left(1+c_{1} b / \beta_{0}\right)}\left(\frac{b}{\beta_{0}}\right)\right. \\
& \left.+\sum_{n=2}^{\infty} \frac{\left(\tilde{d}_{n}-c_{1} \tilde{d}_{n-1}\right)}{\left(1+c_{1} b / \beta_{0}\right)\left(2+c_{1} b / \beta_{0}\right) \cdots\left(n+c_{1} b / \beta_{0}\right)}\left(\frac{b}{\beta_{0}}\right)^{n}\right\} .
\end{aligned}
$$

Here, $\tilde{d}_{j}=d_{j}\left(\mu^{2} / Q^{2} ; c_{2}, \ldots, c_{j}\right)$ with $\mu^{2}=Q^{2}, c_{k}=c_{1}^{k}(k=2, \ldots, j)$. We refer to the Appendix for details. Expanding each term on the right-hand side in powers of $b$ then yields the expansion of $\bar{D}(b ; \tilde{c})$ around $b=0$. Here we can see again that in the large- $\beta_{0}$ approximation $\left(c_{1} \rightarrow+0\right)$ the above expansion reduces to the expansion (21) of the ordinary Borel transform $\widetilde{D}(b ; 1, \ldots)$ times the factor $\exp \left[\left(\tilde{c}-\tilde{d}_{1}\right) b / \beta_{0}\right]$.

The ordinary Borel transform $\widetilde{D}(b)$ is known to have singularities at $b=-1,-2, \ldots$ (UV renormalons) and at $b=2,3, \ldots$ (IR renormalons). The renormalon resummation of $D\left(Q^{2}\right)$ and of the hadronic $\tau$ decay width in the large- $\beta_{0}$ limit has been performed in Refs. [52 54]. The IR renormalons on the contour of the Borel integration (20) cause ambiguities, above all the leading IR renormalon $(b=2)$. The singularity of $D(b)$ at $b=n$ has the form $1 /(1-b / n)^{2+\gamma_{n}^{\prime}+n c_{1} / \beta_{0}}$ for $n \geq 3$, and $1 /(1-b / 2)^{1+\gamma_{2}^{\prime}+2 c_{1} / \beta_{0}}$ at $b=2$, where $\gamma_{n}^{\prime}$ is the one-loop anomalous dimension of the operators corresponding to the $1 / Q^{2 n}$-terms in the operator product expansion (OPE) of the Adler function $D\left(Q^{2}\right)$. In the case $n=2$, it is known that $\gamma^{\prime}=0$ [55]. Thus, at the leading IR renormalon we have $\widetilde{D}(b) \propto 1 /(1-b / 2)^{1+\nu}$, with $\nu=2 c_{1} / \beta_{0}\left(=1.580\right.$, when $\left.n_{f}=3\right)$.

On the other hand, the modified Borel transform $\bar{D}(b)$ in Eq. (23) has the (IR and UV) renormalon singularities at the same locations as $\widetilde{D}(b)$, but with simpler powers [41,42 the IR renormalon singularities are of the form $1 /(1-b / n)^{2+\gamma_{n}^{\prime}}$ for $n \geq 3$, and $1 /(1-b / 2)^{1}$ for the leading IR singularity' [see Eq. (A.13) in the Appendix, with $\kappa=n$ ]. Therefore, we can define a new function $\bar{R}(b ; \tilde{c})$

4 This is true even when the mtH RSch is abandoned and the integral transformation (cf. Appendix) becomes considerably more complicated - as follows from the considerations of Ref. [42]. 


$$
\bar{R}(b ; \tilde{c}) \equiv(1-b / 2) \bar{D}(b ; \tilde{c}),
$$

which has a considerably softened singularity at $b=2$ (cut instead of pole). This function can be determined by resummations of the TPS of $\bar{R}(b ; \tilde{c})$ (cf. also Ref. [15]). The latter TPS is known to the same order as $\bar{D}(b ; \tilde{c})$ - see Eq. (25): $d_{1}^{(0)}$ and $d_{2}^{(0)}\left(\Leftrightarrow \tilde{d}_{1}, \tilde{d}_{2}\right)$ are known exactly, and $d_{3}^{(0)}\left(\Leftrightarrow \tilde{d}_{3}\right)$ is known approximately. The same is true for the modified functions $\bar{R}_{j}(b ; \tilde{c})(j=1,2,3)$ discussed below, Eqs. (27)-(30).

The function $\bar{D}(b ; \tilde{c})$ as defined in Eq. (23) has additional singularities, as seen from the expansion (25): 1.) it is nonanalytic at $b=0$, due to the (finite) factor $\left(c_{1} b / \beta_{0}\right)^{c_{1} b / \beta_{0}} ; 2$.) it has additional (spurious) poles at $b=-\beta_{0} / c_{1}\left(\approx-1.266\right.$ when $\left.n_{f}=3\right), b=-2 \beta_{0} / c_{1}$, etc. In approximate numerical evaluations of $D\left(Q^{2}\right)$ and $r_{\tau}$ via $\bar{D}(b ; \tilde{c})$, such singularities may have disturbing effects unless they are sufficiently far away from the origin. Therefore, we can define the following variants which will be used in (approximate) resummations

$$
\begin{aligned}
\bar{R}_{1}(b ; \tilde{c}) & =(1-b / 2)\left(c_{1} b / \beta_{0}\right)^{-c_{1} b / \beta_{0}} \Gamma\left(1+c_{1} b / \beta_{0}\right) \mathrm{e}^{c_{1} b / \beta_{0}} \bar{D}(b ; \tilde{c}) \\
& =(1-b / 2) \exp \left[\left(\tilde{c}+c_{1}-\tilde{d}_{1}\right) b / \beta_{0}\right]\left\{1+\frac{\left(\tilde{d}_{1}-c_{1}\right)}{\left(1+c_{1} b / \beta_{0}\right)}\left(\frac{b}{\beta_{0}}\right)+\ldots\right\}, \\
\bar{R}_{2}(b ; \tilde{c}) & =\bar{R}_{1}(b ; \tilde{c}) \frac{\left(1+c_{1} b / \beta_{0}\right)}{\left(2+c_{1} b / \beta_{0}\right)}, \\
\bar{R}_{3}(b ; \tilde{c}) & =\bar{R}_{1}(b ; \tilde{c}) \frac{\left(1+c_{1} b / \beta_{0}\right)\left(2+c_{1} b / \beta_{0}\right)}{\left(3+c_{1} b / \beta_{0}\right)^{2}} .
\end{aligned}
$$

We recall that $\bar{R}_{j}(b ; \tilde{c})=\exp \left(\tilde{c} b / \beta_{0}\right) \bar{R}_{j}(b ; 0)$. In the above expressions, we restored analyticity at $b=0$ by factoring out, instead of the factor $\left(c_{1} b / \beta_{0}\right)^{c_{1} b / \beta_{0}}$, the combination $\left(c_{1} b / \beta_{0}\right)^{c_{1} b / \beta_{0}} \mathrm{e}^{-c_{1} b / \beta_{0}} / \Gamma\left(1+c_{1} b / \beta_{0}\right)$. Our main motivation for this lies in the following: the factor $\left(c_{1} b / \beta_{0}\right)^{c_{1} b / \beta_{0}}$ is increasing extremely fast with increasing $b$, while the aforementioned combination has weak $b$-dependence

$$
\frac{\left(c_{1} b / \beta_{0}\right)^{c_{1} b / \beta_{0}} \mathrm{e}^{-c_{1} b / \beta_{0}}}{\Gamma\left(1+c_{1} b / \beta_{0}\right)}=\frac{1}{\sqrt{2 \pi c_{1} b / \beta_{0}}} \text { when } \quad b \rightarrow \infty .
$$

Hence, functions $R_{j}(b ; \tilde{c})$ behave at large $b$ roughly as $R(b ; \tilde{c})$, or $\bar{D}(b ; \tilde{c})$,

$$
\bar{R}_{j}(b ; \tilde{c}) \sim(1-b / 2) \sqrt{2 \pi c_{1} b / \beta_{0}} \bar{D}(b ; \tilde{c}) \quad \text { when } b \rightarrow \infty,
$$

i.e., they neither decrease nor increase violently. Therefore, any approximate resummation method will have better chance when applied to them than to an extremely fast increasing or decreasing version. On the other hand, if we just factored out the factor $\left(c_{1} b / \beta_{0}\right)^{c_{1} b / \beta_{0}}$, the resulting function, though analytic at $b=0$, would at large $b$ decrease violently, as $\sim\left(c_{1} b / \beta_{0}\right)^{-c_{1} b / \beta_{0}} \bar{D}(b ; \tilde{c})$.

The function $R_{1}(b ; \tilde{c})$ has spurious (unphysical) poles at $b=-\beta_{0} / c_{1},-2 \beta_{0} / c_{1}, \ldots \approx \approx$ $-1.261,-2.53$, when $n_{f}=3$ ). But the function $R_{2}$ has a possible advantage over $R_{1}$ in resummations, since it has no spurious (nonphysical) pole at $b=-\beta_{0} / c_{1}$, and $R_{3}$ has no such poles at $b=-\beta_{0} / c_{1}$ and $-2 \beta_{0} / c_{1}$.

Since the coefficients $d_{1}^{(0)}$ and $d_{2}^{(0)}\left(\Rightarrow \tilde{d}_{1}, \tilde{d}_{2}\right)$ of the (massless) Adler function are known, the power expansion of $\bar{R}_{j}(b ; \tilde{c})$ is known to the next-to-next-to-leading order (NNLO, including the term $\sim b^{2}$ ) via Eqs. (25)-(30). In a previous work [22], we presented an argument, 
via a bilocal expansion of the Borel amplitude $\widetilde{D}(b)$, that $d_{3}^{(0)} \approx 25 \pm 5$. We also discussed there the estimates of $d_{3}^{(0)}$ presented by other authors, and concluded that the following estimate is rather safe

$$
d_{3}^{(0)}\left[\equiv d_{3}\left(\mu^{2}=Q^{2} ; \overline{\mathrm{MS}}\right)\right]=25 \pm 10
$$

We will use the above values which allow us to obtain the power series of $\bar{R}_{j}(b ; \tilde{c})$ up to $\mathrm{N}^{3} \mathrm{LO}\left(\sim b^{3}\right)$.

\section{RESUMMATION PROCEDURE}

We will apply summations to the $\mathrm{N}^{3} \mathrm{LO}$ truncated power series (TPS) of the functions $\bar{R}_{j}(b ; \tilde{c})$. However, in order to obtain the (resummed) values of the massless QCD observable $r_{\tau}$ (14), we have to perform first the complex momentum contour integration (16), with the massless Adler function there having the integral form (23) in terms of the invariant Borel transform $\bar{D}\left(b ; Q^{2}=m_{\tau}^{2} e^{\mathrm{i} y}\right)$, i.e., in terms of the related functions $\bar{R}_{j}(27)-(30)$. This angular $y$-integration can be performed exactly, because the $y$-dependence of the invariant $\rho_{1}(24)$ appearing in Eq. (23) is simple

$$
\begin{gathered}
\rho_{1}\left(m_{\tau}^{2} e^{\mathrm{i} y}\right)=\rho_{1}\left(m_{\tau}^{2}\right)+\mathrm{i} \beta_{0} y \quad \Rightarrow \\
r_{\tau}=\frac{1}{\pi \beta_{0}} \operatorname{Re}\left[\int_{0-i \varepsilon}^{\infty-i \varepsilon} d b \exp \left[-\frac{\left(\rho_{1}\left(m_{\tau}^{2}\right)+\tilde{c}\right) b}{\beta_{0}}\right] \frac{\sin (\pi b)}{b(1-b)(1-b / 3)(1-b / 4)} \bar{D}(b ; \tilde{c})\right], \\
=\frac{1}{\pi \beta_{0}} \operatorname{Re}\left\{\int_{0-i \varepsilon}^{\infty-i \varepsilon} d b \exp \left[-\frac{\left(\rho_{1}\left(m_{\tau}^{2} ; a_{0}\right)+\tilde{c}\right) b}{\beta_{0}}\right] \frac{\sin (\pi b)}{b(1-b)(1-b / 2)(1-b / 3)(1-b / 4)}\right. \\
\left.\quad \times \frac{\left(c_{1} b / \beta_{0}\right)^{c_{1} b / \beta_{0}} e^{-c_{1} b / \beta_{0}}}{\Gamma\left(1+c_{1} b / \beta_{0}\right)} f_{j}(b) \bar{R}_{j}(b ; \tilde{c})\right\},
\end{gathered}
$$

where

$$
f_{j}(b)=\left\{\begin{array}{ll}
1 & \text { if } j=1, \\
\left(2+c_{1} b / \beta_{0}\right) /\left(1+c_{1} b / \beta_{0}\right) & \text { if } j=2, \\
\left(3+c_{1} b / \beta_{0}\right)^{2} /\left(1+c_{1} b / \beta_{0}\right) /\left(2+c_{1} b / \beta_{0}\right) & \text { if } j=3 .
\end{array}\right\}
$$

The integration contour in Eqs. (35)-(36) is chosen slightly below (or above) the positive axis, in order to avoid possible singularities of the integrand on the positive axis. When knowing reasonably well the RScl- and RSch-invariant functions $R_{j}(b)$ in Eq. (36), e.g. via resummations as discussed below, the massless QCD observable $r_{\tau}$ becomes an expression whose value depends uniquely on the value of the QCD coupling parameter, e.g. on $\alpha_{s}\left(m_{\tau}^{2} ; \overline{\mathrm{MS}}\right) \equiv \pi a_{0}$. This dependence originates from the known dependence of the invariant $\rho_{1}\left(m_{\tau}^{2}\right)$ in the exponent in Eq. (36), the latter being determined by an integrated version of the RGE, called also the (unsubtracted) Stevenson equation [2]

$$
\begin{aligned}
\rho_{1}\left(m_{\tau}^{2} ; a_{0}\right) & \equiv-d_{1}^{(0)}+\beta_{0} \ln \frac{m_{\tau}^{2}}{\widetilde{\Lambda}} \\
& =-d_{1}^{(0)}+\frac{1}{a_{0}}+c_{1} \ln \left(\frac{c_{1} a_{0}}{1+c_{1} a_{0}}\right)+\int_{0}^{a_{0}} d x\left[\frac{1}{x^{2}\left(1+c_{1} x\right)}+\frac{\beta_{0}}{\beta_{\overline{\mathrm{MS}}}(x)}\right],
\end{aligned}
$$


where the perturbation expansion of the last term is given via the definition (11) of the $\beta$-function

$$
\beta_{\overline{\mathrm{MS}}}(x) / \beta_{0}=-x^{2}\left(1+c_{1} x+c_{2}^{\overline{\mathrm{MS}}} x^{2}+c_{3}^{\overline{\mathrm{MS}}} x^{3}+\cdots\right) .
$$

Here, the $\overline{\mathrm{MS}}$ coefficients are functions of the number of active quark flavors $n_{f}$ and are known up to the $\mathrm{N}^{3} \mathrm{LO}\left(c_{3}^{\overline{\mathrm{MS}}}\right.$, four-loop) [56]. The number of active quark flavors is assumed to be here $n_{f}=3$, because the scale of the process is $\sqrt{\left|Q^{2}\right|}=m_{\tau}(\approx 1.777 \mathrm{GeV})$.

We can note from Eq. (36) that ambiguity of integration over $b$ in $r_{\tau}$ at the first infrared renormalon singularity of $\bar{D}(b)$ at $b=2$ is suppressed, because the factor $\sin (\pi b)$ has a zero there. This is similar to the one-loop approximation in the approach with the ordinary Borel transform [16,57. However, here the absence of the ambiguity is not due to an approximation, it is exact and due to the discussed RScl-invariant Borel approach.

Although the ambiguity at $b=2$ is suppressed in the Borel-type of integration (36), we wish to emphasize that it is nonetheless very important to factor out the leading renormalon factor $1 /(1-b / 2)$ there [according to Eq. (26)]. This is so because any resummation of a $\operatorname{TPS}(b)$ represents also a quasianalytic continuation of the corresponding function into the region away from the origin, and such continuation is of a better quality when there are as few singularities near the origin as possible. The functions which we will resum in Eq. (36) are $\bar{R}_{j}(b ; \tilde{c})$, i.e., the functions which have their original pole singularity at $b=2$ factored away according to Eq. (26). The remaining singularity in $\bar{R}_{j}(b ; \tilde{c})$ is then significantly weaker, it is a cut of the type $\sim \ln (1-b / 2$ ) [see Eq. (A.13) with $\alpha=1$ and $\kappa=2$, in conjunction with Eq. A.20)]. In this way, in the relatively wide region $b \lesssim 2$ we can achieve reasonably good values of the integrand of Eq. (36), which leads then to good predictions for $r_{\tau}$ via Eq. (36) as functions of $\alpha_{s}^{\overline{\mathrm{MS}}}\left(m_{\tau}^{2}\right)$. If we did not factor out the mentioned singularity, the obtained (resummed) values would definitely lead to bad values of the integrand at $b \sim 2$, the fact which would influence the predicted values of $r_{\tau}$ and thus of $\alpha_{s}^{\overline{\mathrm{MS}}}\left(m_{\tau}^{2}\right)$. The numerical importance of factoring out the leading IR renormalon singularity of the Borel transforms of the Adler function at $b=2$ before doing resummation was pointed out in our previous work [22], which involved ordinary Borel transforms. There we showed that the predicted values of $\alpha_{s}^{\overline{\mathrm{MS}}}\left(m_{\tau}^{2}\right)$ from $r_{\tau}$ depend crucially on whether this factoring-out procedure has been performed.

The functions $\bar{R}_{j}(b ; \tilde{c})$ in Eq. (36), whose TPS's we want to resum via methods of quasianalytic continuation, have the singularities near the origin which are negative $($ at $b=-1$ and lower). The nearest singularity on the positive axis is at $b=3$. At $b=2\left(\mathrm{IR}_{2}\right)$ there is a weaker logarithmic singularity. The negative singularities near the origin constrain the convergence radius of the perturbation (power) series of $\bar{R}_{j}(b ; \tilde{c})$ 's to $r=1$, and thus represent a possible hindering element to efficient resummations. In our previous work [22] we proposed how to extend the convergence radius up to $\mathrm{IR}_{2}$ - by either of the following two conformal transformations $w=w(b)$ :

$$
w_{3}(b)=\frac{\sqrt{1+b}-\sqrt{1-b / 3}}{\sqrt{1+b}+\sqrt{1-b / 3}}, \quad w_{4}(b)=\frac{\sqrt{1+b}-\sqrt{1-b / 4}}{\sqrt{1+b}+\sqrt{1-b / 4}} .
$$

Conformal transformation $w_{3}(b)$ maps all the renormalon singularities to the unit circle in the $w$-plane, except for the first IR renormalon which is mapped to $w_{3}(b=2)=1 / 2$. We further 
note that $w_{3}(b=3)=1$, and $w_{3}(b=-1)=-1$. Furthermore, all the spurious singularities $b=-n \beta_{0} / c_{1}(<-1.265)$ are also sent to the unit circle. Conformal transformation $w_{4}(b)$ does the same thing, except that the first two IR renormalon singularities are both inside the unit circle: $w_{4}(b=2) \approx 0.42, w_{4}(b=3)=0.6$. We further note that $w_{4}(b=4)=1$, and $w_{4}(b=-1)=-1$. The inverse transformations are

$$
b\left(w_{3}\right)=\frac{3 w_{3}}{\left(1-w_{3}+w_{3}^{2}\right)}, \quad b\left(w_{4}\right)=\frac{(16 / 5) w_{4}}{\left(1-(6 / 5) w_{4}+w_{4}^{2}\right)},
$$

which are monotonously increasing functions for $0<w_{3}, w_{4}<1$. We can now reexpress the $\left(\mathrm{N}^{3} \mathrm{LO}\right)$ TPS of $\bar{R}_{j}(b ; \tilde{c})$ (in powers of $b$ ) as $\left(\mathrm{N}^{3} \mathrm{LO}\right)$ TPS in powers of $w_{3}$ or $w_{4}$, by simply using the power expansions of Eq. (41). The advantage of using this form of TPS for resummations lies in the fact that the convergence radius for the power series of $\bar{R}_{j}$ is now $r_{w_{3}}=1 / 2$ in the $w_{3}$-plane, and $r_{w_{4}} \approx 0.42$ in the $w_{4}$-plane, and the circle of convergence reaches thus the first IR renormalon singularity $b\left(w_{k}\right)=2(k=3,4)$, in contrast to the case of nontransformed $b$. In this way, the hindering influence of the UV singularities (negative $b$ 's) has been significantly weakened. The mapping $w_{4}(b)$ apparently suppresses even more strongly the influence of the UV singularities than $w_{3}(b)$, but probably less strongly the influence of the NLO IR renormalon singularity $(b=3)$. The final formula for $r_{\tau}$ in this formulation follows directly from the form (36)

$$
\begin{aligned}
r_{\tau}= & \frac{1}{\pi \beta_{0}} \operatorname{Re}\left\{e^{-\mathrm{i} \phi} \int_{0}^{1} d x \frac{d b(w)}{d w} \exp \left[-\frac{\left(\rho_{1}\left(m_{\tau}^{2} ; a_{0}\right)+\tilde{c}\right) b(w)}{\beta_{0}}\right]\right. \\
& \times \frac{\sin (\pi b(w))}{b(w)(1-b(w))(1-b(w) / 2)(1-b(w) / 3)(1-b(w) / 4)} \\
& \left.\times\left.\frac{\left(c_{1} b(w) / \beta_{0}\right)^{c_{1} b(w) / \beta_{0}} e^{-c_{1} b(w) / \beta_{0}}}{\Gamma\left(1+c_{1} b(w) / \beta_{0}\right)} f_{j}(b(w)) \bar{R}_{j}(b(w) ; \tilde{c})\right|_{w=x e^{-\mathrm{i} \phi}}\right\},
\end{aligned}
$$

where $f_{j}(b)$ 's are fiven by Eq. (37), $w$ stands for $w_{3}$ or $w_{4}$, and we integrate actually only up to a $w_{\max }$ corresponding to $b \approx 4$ (for $w_{3}, \phi_{3}=0.50536, w_{3 \max }=\exp \left(-\mathrm{i} \phi_{3}\right)$; for $w_{4}$, $\left.\phi_{4}=0.1 \approx 0, w_{4 \max }=\exp \left(-\mathrm{i} \phi_{4}\right) \approx 1\right)$. This upper bound on $b$ is justified because the contributions from higher $b$ 's are very strongly suppressed due to the exponent in Eq. (42). Strictly speaking, the path in the $w_{3}$-plane should be along the positive axis (below it) from $w_{3}=0$ to $w_{3}=1$, and then along the arc (inner side) of the unit circle between $w_{3}=1$ and $w_{3}=w_{3}(b=4)=\exp \left(-\mathrm{i} \phi_{3}\right)$, as shown in Fig. 1. However, for practical calculations, it is much more convenient to use the integration along the ray $w_{3}=x \exp \left(-\mathrm{i} \phi_{3}\right)(0 \leq x \leq 1)$, as denoted in Eq. (42) and shown in Fig. 1. Both paths give the same answer, since the closed contour in Fig. 1 does not contain any singularities of the integrand. In the case of $w_{4}$, the trick is the same, and we choose $\phi_{4}=0.1$ instead of $\phi_{4}=0$ for the ray (see Fig. 2), in order to avoid any possible problems with numerical instability that would otherwise arise from the too extreme vicinity of the integration path to the possible singularities of the integrand.

At this point, we can thus already regard the $\mathrm{N}^{3} \mathrm{LO}$ TPS of the function $\bar{R}_{j}\left(b\left(w_{k}\right) ; \tilde{c}\right) \equiv$ $\exp \left[\tilde{c} b\left(w_{k}\right) / \beta_{0}\right] \bar{R}_{j}\left(b\left(w_{k}\right) ; 0\right)(k=3,4)$ as a form of resummation of $\bar{R}_{j}$, solely via the mappings (40). Consequently, expressions (42) evaluated using the aforementioned $\mathrm{N}^{3} \mathrm{LO}$ TPS of $\bar{R}_{j}\left(b\left(w_{k}\right) ; \tilde{c}\right)$ can be regarded as our resummed predictions of $r_{\tau} \equiv r_{\tau}\left(a_{0}\right)$, being functions of the mentioned strong QCD couplant $a_{0}$. There is, however, an additional freedom of 
choosing the value of the constant $\tilde{c}$ in Eq. (42). f Since the available series of $\bar{R}_{j}\left(b\left(w_{k}\right) ; \tilde{c}\right)$ is truncated, the results of Eq. (42) will have some unphysical dependence on the value of $\tilde{c}$. In one of our approaches, we will choose the latter value by the principle of minimal sensitivity (PMS), i.e., by the condition

$$
\frac{\partial r_{\tau}\left(a_{0} ; \tilde{c}\right)}{\partial \tilde{c}}=0
$$

when using for $\bar{R}_{j}\left(b\left(w_{k}\right)\right)$ their $\mathrm{N}^{3} \mathrm{LO}$ TPS forms.

We can, however, proceed in a different way. The $\mathrm{N}^{3} \mathrm{LO}$ TPS of $\bar{R}_{j}\left(b\left(w_{k}\right) ; \tilde{c}\right)$ can be further resummed using Padé approximants (PA's) [58]. [] The authors of Ref. [59] presented compelling arguments that combining the conformal transformations with the PA-type of resummations in general leads to significantly improved results, at least when a sufficient number of terms in the power expansion are known. Especially the diagonal or almost diagonal PA's [58,59], in our case $[2 / 1]_{R_{j}}\left(w_{k}\right)$ and $[1 / 2]_{\bar{R}_{j}}\left(w_{k}\right)$, may represent an efficient way of extending the applicability of expressions for $\bar{R}_{j}$ into the region sufficiently far away from the origin (quasianalytic continuation).

However, there is a possibility that PA's do not lead to an improvement. This is sometimes the case when the TPS in question is known to a relatively low order, e.g. to the $\mathrm{N}^{3} \mathrm{LO}$. Since our available TPS's of $\bar{R}_{j}\left(w_{k} ; \tilde{c}\right)(k=3,4)$ are known to the $\mathrm{N}^{3} \mathrm{LO}$ (provided a specific value of $d_{3}^{(0)}$ is taken), we have to find criteria for keeping or rejecting the resulting PA's. The PA $[2 / 1]_{\bar{R}_{j}}\left(w_{k} ; \tilde{c}\right)$ predicts one real singularity of $\bar{R}_{j}\left(b\left(w_{k}\right) ; \tilde{c}\right)$, and $[1 / 2]_{\bar{R}_{j}}\left(w_{k} ; \tilde{c}\right)$ two singularities which can be either real or mutually complex conjugate. Physically, $\bar{R}_{j}\left(b\left(w_{k}\right) ; \tilde{c}\right)$ has the strongest singularities (UV and IR renormalon singularities) at $b\left(w_{k}\right)=-1,-2, \ldots$ and $b\left(w_{k}\right)=3,4, \ldots$ This means that in the case of conformal transformations (40), the singularities of PA's should preferably be at $w_{k}$ values corresponding to $b=-1$ or 3 : $w_{3}^{\text {pole }}=-1$ or $+1 ; w_{4}^{\text {pole }}=-1$ or +0.6 . We will include in our analysis the predictions with those PA's $[2 / 1]_{R_{j}}\left(w_{k}\right)$ whose poles satisfy the latter conditions. For that, we will use our freedom to adjust the value of the constant $\tilde{c}$ in Eq. (42). On the other hand, PA's $[1 / 2]_{\bar{R}_{j}}\left(w_{k} ; \tilde{c}\right)$, which have two poles, only rarely satisfy approximately the aforementioned conditions simultaneously.

Furthermore, in practical calculations, we prefer to use for the $\beta_{\overline{\mathrm{MS}}}(39)$ in the Stevenson's equation (38) the PA $[2 / 3]_{\beta \overline{\mathrm{MS}}}(x)$, particularly because of its reasonable singularity structure $\left(x_{\text {pole }} \approx 0.311\right.$, corresponding to $\left.\alpha_{s}^{\text {pole }} \approx 0.98\right)$. The latter signals the breakdown of perturbative QCD (pQCD), and this choice of $\beta_{\overline{\mathrm{MS}}}(x)$ has been used previously by some of us in Refs. [22,21]. Since the effective energy in the discussed QCD observable $r_{\tau}$ is relatively low $E \sim m_{\tau}<2 \mathrm{GeV}$, we can expect that this choice is not entirely irrelevant numerically.

${ }^{5}$ Variation of $\tilde{c}$ in Eq. (42) corresponds to changing somewhat the numerical procedure used.

${ }^{6} \mathrm{PA}[n / m]_{R}(w)$ to a function $R(w)$ is a ratio of polynomials in $w$ of degree $n$ (numerator) and $m$ (denominator). The power expansion of $[n / m]_{R}(w)$ must reproduce the terms of the power expansion of $R(w)$ up to, and including, the term $\sim w^{n+m}$. PA $[n / m]_{R}(w)$ can be determined if we know the TPS of $R(w)$ up to, and including, the term $\sim w^{n+m}$. PA's $[n / n]$ are called diagonal. 
Later we will comment on how much the results change when employing the $\left(\mathrm{N}^{3} \mathrm{LO}\right)$ TPS for $\beta_{\overline{\mathrm{MS}}}(x)$, instead.

\section{PREDICTIONS FOR $\alpha_{s}$; THEORETICAL UNCERTAINTIES ESTIMATE}

As discussed in the previous section, for a given choice of $a_{0} \equiv \alpha_{s}\left(m_{\tau}^{2} ; \overline{\mathrm{MS}}\right) / \pi$ and of constant $\tilde{c}$, we calculate the observable $r_{\tau} \equiv r_{\tau}\left(a_{0} ; \tilde{c}\right)$ (42), using for $\bar{R}_{j}\left(b\left(w_{k}\right) ; \tilde{c}\right)$ one of the variants (27)-(30) $(j=1,2,3)$, and one of the two conformal transformations (41) $(k=3,4)$, and for the resummation of $\bar{R}_{j}\left(b\left(w_{k}\right)\right)$ either the $\mathrm{N}^{3} \mathrm{LO}$ TPS or [2/1] PA. We thus use, at given $\tilde{c}$, altogether $3 \times 2 \times 2=12$ ways of calculating $r_{\tau}$ as function of $a_{0} \equiv \alpha_{s}\left(m_{\tau}^{2}, \overline{\mathrm{MS}}\right) / \pi$. The value $\tilde{c}$ is adjusted as described at the end of the previous section: (a) according to the PMS (43) when using $\mathrm{N}^{3} \mathrm{LO}$ TPS for $\bar{R}_{j}\left(b\left(w_{k}\right)\right)$; (b) according to the pole requirements $w_{3}^{\text {pole }}=-1$ or $+1\left(w_{4}^{\text {pole }}=-1\right.$ or +0.6$)$ when using $\mathrm{PA}[2 / 1]_{\bar{R}_{j}}\left(w_{k}\right)$ for $\bar{R}_{j}\left(b\left(w_{k}\right)\right)$. We repeat the analysis for three choices of the $\mathrm{N}^{3} \mathrm{LO}$ coefficient $d_{3}^{(0)}$ (33) of the Adler function: $d_{3}^{(0)}=25,15,35$ In the central case of $d_{3}^{(0)}=25$, and for $r_{\tau}$ close to the central measured value $r_{\tau}=0.1960$ (18), we display the relevant numerical results in Table $\mathbb{\mathbb { L }}$. Displayed are the predictions of various approximants for the input values $\alpha_{s}\left(m_{\tau}^{2} ; \overline{\mathrm{MS}}\right)=0.325$ and 0.326 . The first 14 entries are predictions of Eq. (42) when using PA's $[2 / 1]_{\bar{R}_{j}}\left(w_{k}\right)(j=1,2,3$; $k=3,4$ ), where $\tilde{c}$ was adjusted so that these PA's yield the aforementioned pole values of the leading UV or subleading IR renormalon pole. The next six entries are predictions when using $\mathrm{N}^{3} \mathrm{LO}$ TPS for $\bar{R}_{j}\left(b\left(w_{k}\right)\right)$ in Eq. (42) with $\tilde{c}$ adjusted so that the PMS principle (43) is satisfied (local maximum). The last entry contains the arithmetic average $\bar{r}_{\tau}$ of all 20 predictions, for the aforementioned two values of $\alpha_{s}\left(m_{\tau}^{2} ; \overline{\mathrm{MS}}\right)$. From that entry, we deduce that the central measured value $r_{\tau}=0.1960$ (18) is achieved by this arithmetic average $\bar{r}_{\tau}$ at $\alpha_{s}\left(m_{\tau}^{2} ; \overline{\mathrm{MS}}\right)=0.3254$. The uncertainty of the prediction due to resummation method ("truncation" error) can be estimated by comparing the aforementioned prediction with the prediction which differs the most from it, i.e., with the prediction using the PA $[2 / 1]_{\bar{R}_{j}}\left(w_{k}\right)$ with $j=3, k=4$ and $\tilde{c}=1.34$ (see the 14th entry of Table $\mathbb{\mathbb { I }}$ ). This prediction differs from the aforementioned one by $\left|\delta \alpha_{s}\right|_{\text {tr. }} \approx 0.0024$.

Repeating the very same calculations (at the same values of $\tilde{c}$ ) for the correspondingly higher and lower input values of $\alpha_{s}\left(m_{\tau}^{2} ; \overline{\mathrm{MS}}\right)$, we obtain the corresponding predictions of $\alpha_{s}\left(m_{\tau}^{2} ; \overline{\mathrm{MS}}\right)$ for the upper and lower bounds of the measured values of $r_{\tau}$ (18) by demanding that the arithmetic average $\bar{r}_{\tau}$ be equal to those upper and lower bounds. This procedure then results in the prediction

$$
\alpha_{s}^{\overline{\mathrm{MS}}}\left(m_{\tau}^{2}\right)=0.3254 \pm 0.0060_{\mathrm{exp} .} \pm 0.0060_{\mathrm{EW}} \pm 0.0052_{\mathrm{CKM}} \pm 0.0024_{\mathrm{tr} .} \quad\left(d_{3}^{(0)}=25\right) .
$$

It is gratifying that in the case of $d_{3}^{(0)}=25$ so many PA cases give physically acceptable pole structure, and that the predictions for $\alpha_{s}^{\overline{\mathrm{MS}}}\left(m_{\tau}^{2}\right)$ of the aforementioned 20 different approaches differ from each other only little - they differ by at most 0.0024 from the prediction of their total average. If confining ourselves to just one conformal transformation, the central value changes by only \pm 0.0001 ( 0.3255 for $k=3$; 0.3253 for $k=4)$. If using only the six entries from the approach with the PMS, the central value is 0.3259 . If using only the

entries with $w_{k}^{\text {pole }} \approx-1$, the central value is 0.3258 . If using only the entries with $w_{3}^{\text {pole }} \approx+1$ and $w_{4}^{\text {pole }} \approx+0.6$, the central value changes to 0.3244 . 
Further, for most of the approximants of the first eight entries of Table [ (except those with $j=1$ ), when using the same values of $\tilde{c}$ but using PA's [1/2] instead of [2/1] for $\bar{R}_{j}\left(w_{k}\right)$, the predictions $r_{\tau}$ differ from those of $[2 / 1]$ by no more than 0.0005 , and the predictions of $\alpha_{s}\left(m_{\tau}^{2}\right)$ also by no more than 0.0005 . In these cases of good agreement, the poles $w_{k}^{\text {pole }}$ of the PA's [1/2] do not lie deep inside the $b\left(w_{k}\right)$-intervals $[-1,+3]$. In most of the cases when the disagreement is larger, at least one of the poles of the [1/2] falls deep inside these intervals, often even within the intervals $[-0.5,+2]$. This offers us an additional evidence that our requirement that the poles of [2/1] be either at $b\left(w_{k}\right)=-1$ (leading UV renormalon pole) of $b\left(w_{k}\right)=+3$ (next-to-leading IR renormalon pole) was reasonable.

In order to obtain the uncertainties of the prediction due to the uncertainty $d_{3}^{(0)}=25 \pm 10$ around the central value $d_{3}^{(0)}=25$, we repeat the procedure for the case of $d_{3}^{(0)}=15$ and $d_{3}^{(0)}=35$.

In the case $d_{3}^{(0)}=15$, the situation is very similar to the afore-discussed case of $d_{3}^{(0)}=25$. We obtain six entries when we require $w_{k}^{\text {pole }}=-1$; three entries each when $w_{3}^{\text {pole }}=+1$ and $w_{4}^{\text {pole }}=+0.6$; six entries when applying the PMS condition (43) with $\mathrm{N}^{3} \mathrm{LO}$ TPS for $\bar{R}_{j}\left(b\left(w_{k}\right)\right)$ - see Table [1], when two choices $\alpha_{s}\left(m_{\tau}^{2} ; \overline{\mathrm{MS}}\right)=0.331$ and 0.332 are made. The arithmetic average $\bar{r}_{\tau}$ of all these 18 entries is then equal to the central measured value (18) $r_{\tau}=0.1960$ when $\alpha_{s}\left(m_{\tau}^{2} ; \overline{\mathrm{MS}}\right)=0.3299$, i.e., the value higher by 0.0045 than the corresponding central prediction of Eq. (44) of the case $d_{3}^{(0)}=25$.

In the case $d_{3}^{(0)}=35$, the numerical situation is less favorable. None of the approaches with PA's $[2 / 1]_{\bar{R}_{j}}\left(w_{k}\right)$ have acceptable solutions under the requirement $w_{k}^{\text {pole }}=-1$. Such poles occur at $\tilde{c}<-2$, but the corresponding predictions of $r_{\tau}$ there are quite unstable under the variation of $\tilde{c}$. In two cases, acceptable solutions are obtained with the approach with PA's $[2 / 1]_{\bar{R}_{j}}\left(w_{k}\right)$ when we require $w_{3}^{\text {pole }}=+1$ or $w_{4}^{\text {pole }}=+0.6-$ see the first two entries of Table [II. The approach with the PMS (43), when using $\mathrm{N}^{3} \mathrm{LO}$ TPS for $\bar{R}_{j}\left(b\left(w_{k}\right)\right)$, appears to be more difficult as well; the derivatives $\partial r_{\tau} / \partial \tilde{c}$ are never zero, but are negative for all reasonable values of $\tilde{c}$; nonetheless, these slopes have the smallest negative values at specific values of $\tilde{c}$ - see the corresponding six entries in Table III. Taking the arithmetic average $\bar{r}_{\tau}$ of the eight entries of Table $\mathbb{\Pi 1}$, we infer that the arithmetic average achieves the central measured value (18) $r_{\tau}=0.1960$ for the value $\alpha_{s}\left(m_{\tau}^{2} ; \overline{\mathrm{MS}}\right)=0.3194$, which is lower by 0.0060 than the corresponding central prediction of Eq. (44) of the case $d_{3}^{(0)}=25$.

From the above considerations we infer that the uncertainty of the prediction of $\alpha_{s}\left(m_{\tau}^{2} ; \overline{\mathrm{MS}}\right)$ due to the uncertainty (33) of $d_{3}^{(0)}$ is \pm 0.0060 .

There is yet another theoretical uncertainty involved in the prediction (44), connected with the choice of the renormalization scheme (RSch). As shown in the Appendix, when taking a RSch different from the modified ' $t$ Hooft $(\mathrm{mtH}) \operatorname{RSch} c_{k}=c_{1}^{k}(k=2,3, \ldots)$, the integral transformations involved become more complicated. Then we end up with, instead of the simpler formula (42), the more complicated one (A.22). We note that the leading RSch parameter $c_{2}$ has the values $c_{2} \approx 3.16 ; 4.47 ; 6.58 ; 5.24$ in the $\mathrm{mtH}, \overline{\mathrm{MS}}$, principle of minimal sensitivity (PMS) [2], and the effective charge (ECH) [3] RSch's (where we take $n_{f}=3$; and the PMS and ECH RSch's refer to the $\mathrm{N}^{3} \mathrm{LO}$ TPS of the Adler function). Therefore, we estimate as a characteristic deviation from $c_{2}=c_{1}^{2} \approx 3.16$ the value $c_{2} \approx c_{1}^{2}+3.4$. We then perform the analysis in the RSch with the latter value of $c_{2}$ (all the other $c_{k}$ 's unchanged), using formula (A.22). We use the RScl parameter value $\xi^{2} \equiv \mu^{2} / Q^{2}=1\left[Q^{2}=m_{\tau}^{2} \exp (\mathrm{i} y)\right]$, 
for $d_{3}^{(0)}$ we use the central value $d_{3}^{(0)}=25$, and for the $\beta$-functions we use again the [2/3] PA form. The results of the analysis are written in Table $\mathbb{} \nabla$. The variation of the parameter $\tilde{c}$ allowed us to obtain the desired locations of the poles only in a few cases. The arithmetic average values $\bar{r}_{\tau}$ of Table $\mathbb{I V}$ reach the central value prediction $\bar{r}_{\tau}=0.1960$ when the the coupling is $\alpha_{s}\left(m_{\tau}^{2} ; \overline{\mathrm{MS}}\right)=0.3285$, which is by 0.0031 higher than the central value in Eq. (44). Concerning the change of the next-to-leading RSch parameter $c_{3}$, we note that $c_{3} \approx 5.6 ; 21.0 ; 36.8 ; 16.1$ for the $\mathrm{mtH}, \overline{\mathrm{MS}}$, PMS and ECH RSch's. Therefore, we choose a characterictic deviation of $c_{3}$ from the $\mathrm{mtH}$ value $c_{3}=c_{1}^{3} \approx 5.6$ to be $c_{3}=c_{1}^{3}+31$. Completely analogous analysis as in the case of the $c_{2}$-deviation leads to the results of Table $\nabla$. The arithmetic average values $\bar{r}_{\tau}$ in Table $\bar{\nabla}$ reach the central value $\bar{r}_{\tau}=0.1960$ for the coupling $\alpha_{s}\left(m_{\tau}^{2} ; \overline{\mathrm{MS}}\right)=0.3274$, which is by 0.0020 higher than the central value in Eq. (44). Therefore, adding the deviations 0.0031 and 0.0020 in quadrature gives the estimated uncertainty due to the changes of the RSch to be \pm 0.0037 .

We can thus add to the prediction (44) the discussed uncertainties due to the variation of $d_{3}^{(0)}$ and of the RSch, resulting in our final prediction

$$
\begin{aligned}
\alpha_{s}^{\overline{\mathrm{MS}}}\left(m_{\tau}^{2}\right)= & 0.3254 \pm 0.0060_{\text {exp. }} \pm 0.0060_{\mathrm{EW}} \pm 0.0052_{\mathrm{CKM}} \\
& \pm 0.0060_{\delta d_{3}} \pm 0.0037_{\mathrm{RSch}} \pm 0.0024_{\mathrm{tr} .}, \\
= & 0.3254 \pm 0.0060_{\text {exp. }} \pm 0.0079_{\mathrm{EW}+\mathrm{CKM}} \pm 0.0074_{\mathrm{th} .} .
\end{aligned}
$$

In the last line, we added the corresponding uncertainties in quadrature; the combined uncertainty due to the uncertainty of $d_{3}^{(0)}$ (33), the resummation ("truncation") uncertainty, and the RSch uncertainty we call the theoretical (th.) uncertainty. This combined uncertainty is comparable with the two other uncertainties in Eq. (46). If we use for the $\overline{\mathrm{MS}}$ $\beta$-function in Eq. (38) the $\mathrm{N}^{3} \mathrm{LO}$ TPS form instead of the $[2 / 3]_{\beta \overline{\mathrm{MS}}}(x)$ PA form, we obtain, in a way completely analogous to that described in Table $\mathbb{\mathbb { L }}$, the central value prediction $\alpha_{s}\left(m_{\tau}^{2} ; \overline{\mathrm{MS}}\right)=0.3245$. This is by 0.0009 lower than the central value prediction in Eqs. (45)(46), indicating that those nonperturbative effects which originate from the behavior of the $\beta$-function are not strong. The fact that we used in the Borel integral a finite $b(w)_{\max }(=4)$ does not influence the results. Namely, if we increase this quantity to $b(w)_{\max }=5$ [corresponding for $w_{3}$ to $\phi_{3}=0.64350$ in Eq. (42), and for $w_{4}$ to $\left.\phi_{4}=0.40272\right]$, the predictions for $\alpha_{s}\left(m_{\tau}^{2}\right)$ change by $\sim 10^{-6}$, i.e., insignificantly.

We then RGE-evolved the result (45)-(46) from the scale $\mu=m_{\tau} \approx 1.777 \mathrm{GeV}$ to the scale $M_{\mathrm{z}}=91.19 \mathrm{GeV}$. We used again the aforementioned $[2 / 3]_{\beta \overline{\mathrm{MS}}}(x) \mathrm{PA}$ form of the $\beta_{\overline{\mathrm{MS}}}$ function, which is based on the known four-loop $\mathrm{N}^{3} \mathrm{LO}$ TPS form of $\beta_{\overline{\mathrm{MS}}}$ 56. Therefore, we employed the corresponding three-loop matching conditions 60 for the flavor thresholds. The matching was performed at $\mu\left(N_{f}\right)=\kappa m_{q}\left(N_{f}\right)$ with the choice $\kappa=2$, where $\mu\left(N_{f}\right)$ is the scale above which $N_{f}$ flavors are assumed active, and $m_{q}\left(N_{f}\right)$ is the running quark mass $m_{q}\left(m_{q}\right)$ of the $N_{f}$ 'th flavor. We further assumed $m_{c}\left(m_{c}\right)=1.25 \mathrm{GeV}$ and $m_{b}\left(m_{b}\right)=4.25$ $\mathrm{GeV}$ 33. We thus obtain from Eqs. (45)-(46)

$$
\begin{aligned}
\alpha_{s}^{\overline{\mathrm{MS}}}\left(M_{\mathrm{z}}^{2}\right) & =0.1192 \pm 0.0007_{\text {exp. }} \pm 0.0010_{\mathrm{EW}+\mathrm{CKM}} \pm 0.0009_{\text {th. }} \pm 0.0003_{\text {evol. }} \\
& =0.1192 \pm 0.0015
\end{aligned}
$$

In Eq. (48), we added all the uncertainties in quadrature. In Eq. (47), we included the uncertainties due to the RGE evolution, which come primarily from varying $\kappa$ from 1.5 to 
3 , and from varying the quark masses $m_{c}\left(m_{c}\right)=1.25 \pm 0.10 \mathrm{GeV}$ and $m_{b}\left(m_{b}\right)=4.25 \pm 0.15$ $\mathrm{GeV}$ (see Ref. [22] for more details).

If we repeat the calculation of $\alpha_{s}\left(m_{\tau}^{2}\right)$ and $\alpha_{s}\left(M_{\mathrm{z}}^{2}\right)$ with the $\mathrm{N}^{3} \mathrm{LO}$ TPS $\beta_{\overline{\mathrm{MS}}}$ function [in Eq. (38) and in the RGE evolution from $m_{\tau}^{2}$ to $\left.M_{\mathrm{z}}^{2}\right]$, instead of the used PA $[2 / 3]_{\beta \overline{\mathrm{MS}}}(x)$, the central value prediction $\alpha_{s}\left(M_{\mathrm{z}}^{2} ; \overline{\mathrm{MS}}\right)=0.1192$ remains unchanged up to the displayed digits. This is so because this change of $\beta_{\overline{\mathrm{MS}}}$ causes the central value prediction of $\alpha_{s}\left(m_{\tau}^{2} ; \overline{\mathrm{MS}}\right)$ to be by 0.0009 lower [as already mentioned after Eqs. (45)-(46)], but then the RGE evolution to $\mu^{2}=M_{\mathrm{z}}^{2}$ with the changed $\beta_{\overline{\mathrm{MS}}}$ pushes the result up, approximately neutralizing the former effect.

In the analysis leading to the results (45)-(48) we assumed that the power-suppressed terms, apart from those from the quark masses, do not contribute to the considered observable $R_{\tau}$, as already emphasized in Sec. [1]. As mentioned in that section, the inclusive $(\mathrm{V}+\mathrm{A})$ fit of the ALEPH Collaboration [27], within their framework, predicted the contributions of the (massless) power-suppressed terms to the canonical observable $r_{\tau}$ to be consistent with zero: $\delta r_{\tau, \mathrm{PS}}=0.000 \pm 0.004$. If we assumed that the latter estimates were valid also in our framework, ] this $\delta r_{\tau, \mathrm{PS}}$ would have to be subtracted from the values given on the right-hand side of (18), resulting in an additional, "PS"-uncertainty, term \pm 0.0040 for the $r_{\tau}$. This would in turn give an additional approximate uncertainty $\pm 0.0041_{\mathrm{PS}}$ in the result (45)-(46) for $\alpha_{s}\left(m_{\tau}^{2}\right)$, and $\pm 0.0005_{\mathrm{PS}}$ in the result (47) for $\alpha_{s}\left(M_{\mathrm{z}}^{2}\right)$. The combined uncertainty \pm 0.0015 for $\alpha_{s}\left(M_{\mathrm{z}}^{2}\right)$ in (48) would increase to \pm 0.0016 . All the central value predictions in (45)-(48) would remain unchanged.

There are at least two indications that the above results (45)-(46) and (47)-(48) are not wrong. In the approach of Ref. [22], which involved ordinary Borel transform of $D\left(Q^{2}\right)$ and where the RScl was fixed according to the (local) PMS and we used the $\overline{M S}$ RSch, the resulting predictions were very similar: $\alpha_{s}\left(m_{\tau}^{2}\right)=0.3267 \pm 0.0062_{\exp .} \pm 0.0082_{\mathrm{EW}+\mathrm{CKM}} \pm$ $0.0073_{\text {meth. }}$ and $\alpha_{s}\left(M_{\mathrm{z}}^{2}\right)=0.1193 \pm 0.0007_{\text {exp. }} \pm 0.0010_{\mathrm{EW}+\mathrm{CKM}} \pm 0.0009_{\text {meth. }} \pm 0.0003_{\text {evol. }}$ $=0.1193 \pm 0.0015$ Especially the latter values are in virtual agreement with Eq. (48).

Yet another indication that the results presented here are correct comes from repeating the entire resummations, but this time without employing the conformal transformations $b=b(w)$. The $\beta$-functions were again taken in the [2/3] PA form. We carried out the resummation with the Borel integration in Eq. (36) again up to $b_{\max }=4$. For simplicity, we fixed this time the $\tilde{c}$ value to $\tilde{c}=0$. In each of the cases $d_{3}^{(0)}=25,15,35$, we excluded from the analysis the approaches with those PA's which give physically unacceptable pole structure, i.e., which have poles well inside the $b$-interval $[-1,3]$. Those of the PA's $[2 / 1]_{\bar{R}_{j}}(b)$ and $[1 / 2]_{\bar{R}_{j}}(b)$ which were not excluded do not contain poles in the $b$-interval $[-0.8,3]$.

In the case $d_{3}^{(0)}=25$, the nonexcluded approaches were those with $[2 / 1]_{\bar{R}_{j}}(b)$ for $j=1,2,3$; and $[1 / 2]_{\bar{R}_{j}}(b)$ for $j=1,2$. Gratifyingly, the $\alpha_{s}$-predictions of these five approaches differ

\footnotetext{
${ }^{7}$ See the discussion in Sec. [1] about the differences between our and ALEPH's framework.

8 The method (meth.) uncertainty in [22] is the combination of uncertainties from $\delta d_{3}^{(0)}$, the truncation (resummation), and the RScl and RSch ambiguities; the method uncertainty there thus corresponds to our theoretical (th.) uncertainty in Eqs. (46) and (47).
} 
from each other only little: $\left|\triangle \alpha_{s}\left(m_{\tau}^{2}\right)\right|<0.0004$. We took the arithmetic average $\bar{r}_{\tau}$ of all five $r_{\tau}$-predictions, and obtained $\alpha_{s}\left(m_{\tau}^{2}\right)=0.3257 \pm 0.0061_{\exp .} \pm 0.0061_{\mathrm{EW}} \pm 0.0053_{\mathrm{CKM}}$ (for $\left.d_{3}^{(0)}=25\right)$.

In the case $d_{3}^{(0)}=35$, the nonexcluded PA approaches were those with $[2 / 1]_{\bar{R}_{j}}(b)$ for $j=1,2$. In addition, we did not exclude the approaches with the N $\mathrm{N}^{3} \mathrm{LO}$ TPS of $\bar{R}_{j}(b)$ for $j=2,3$, because their predictions are close to the aforementioned PA approaches. We then took the arithmetic average $\bar{r}_{\tau}$ of all four $r_{\tau}$ predictions and obtained for the central value $\alpha_{s}\left(m_{\tau}^{2}\right)=0.3191$ [corresponding to the central value $r_{\tau}=0.1960$ in Eq. (18)], which is by 0.0066 lower than the aforementioned central value 0.3257 of the $d_{3}^{(0)}=25$ case.

In the case $d_{3}^{(0)}=15$, the nonexcluded approaches were those with $[2 / 1]_{\bar{R}_{1}}(b)$ and $[1 / 2]_{\bar{R}_{1}}(b)$. Using the arithmetic average $\bar{r}_{\tau}$ of these two $r_{\tau}$ predictions, we obtain the central value $\alpha_{s}\left(m_{\tau}^{2}\right)=0.3293$, which is by only 0.0036 higher than in the $d_{3}^{(0)}=25$ case.

This leads us to the following predictions of our method, when no conformal transformation is used

$$
\begin{aligned}
\alpha_{s}^{\overline{\mathrm{MS}}}\left(m_{\tau}^{2}\right) & =0.3257 \pm 0.0061_{\mathrm{exp} .} \pm 0.0081_{\mathrm{EW}+\mathrm{CKM}} \pm 0.0066_{\delta d_{3}} \\
\alpha_{s}^{\overline{\mathrm{MS}}}\left(M_{\mathrm{z}}^{2}\right) & =0.1192 \pm 0.0007_{\mathrm{exp} .} \pm 0.0010_{\mathrm{EW}+\mathrm{CKM}} \pm 0.0008_{\delta \mathrm{d}_{3}} \pm 0.0003_{\text {evol. }},
\end{aligned}
$$

which is in almost complete agreement with the predictions (45) and (47), obtained by the employment of the two conformal transformations (40). In Eqs. (49) and (50), we did not include the uncertainties due to the resummation (truncation) and due to the RSch ambiguities, because we regard these two predictions only as an additional cross-check of our main predictions and uncertainty estimates (45)-(48).

\section{SUMMARY}

We calculated the hadronic tau decay width $r_{\tau}$ by employing the contour integral form for this quantity in the complex momentum plane (16) and the modified Borel transform for the associated perturbative massless Adler function. By choosing a special renormalization scheme (modified 't Hooft scheme: $c_{k}=c_{1}^{k}, k=2,3, \ldots$ ), the integrand of the modified Borel transform is renormalization scale invariant. In our approach, we explicitly account for the structure of the leading infrared (IR) renormalon of the Adler function via the corresponding ansatz. Further, to accelerate the convergence, i.e., to minimize the resummation (truncation) uncertainties, we employ two different conformal transformations which "map away" all the renormalon singularities, except the leading and subleading IR renormalons, onto the unit circle. The correct location of the leading ultraviolet (UV) renormalon or of the subleading IR renormalon is enforced by employing Padé approximants for the truncated perturbation series of the functions associated with the modified Borel transform. The Borel integration, in this appraoch, turns out to have suppressed renormalon ambiguity for $r_{\tau}$ at the leading IR renormalon singularity, and the ambiguity due to the subleading renormalons is strongly suppressed by the exponent in the Borel integral. We neglect in the observable $r_{\tau}$ all the possible power correction terms (except the dimension $d=4$ quark mass terms), because the results of the ALEPH analysis [27] suggest that such terms are consistent with zero or negligibly small even in our resummation framework. 
Our analysis predicts the values of $\alpha_{s}^{\overline{\mathrm{MS}}}\left(m_{\tau}^{2}\right)$ and $\alpha_{s}^{\overline{\mathrm{MS}}}\left(M_{\mathrm{z}}^{2}\right)$ given in Eqs. (45)- (48). These predictions agree well with the results obtained in our previous analysis [22] of $r_{\tau}$ where we employed the ordinary Borel transforms. The latter transforms have significantly different behavior, expansions and the strengths of the renormalon singularities, and their integrands in the Borel integral are, in contrast to the present approach, renormalization scale dependent. Therefore, our present predictions represent a powerful reconfirmation of the predictions of [22]. We consider this to be important, because analyses of $r_{\tau}$ which do not involve Borel transforms and do not account for the leading renormalon structure of the associated Adler functions [27,61, 10, 19, 62, give predictions for $\alpha_{s}\left(m_{\tau}^{2}\right)$ and $\alpha_{s}\left(M_{\mathrm{z}}^{2}\right)$ which significantly differ from our predictions and significantly differ among themselves, as already emphasized in [22]. On the other hand, if accounting for the renormalon structure via a large $-\beta_{0}$ resummation of the ordinary Borel transform and employing an ECHrelated resummation of $r_{\tau}$, as performed by the authors of Ref. [9], their predicted values $\alpha_{s}^{\overline{\mathrm{MS}}}\left(M_{\mathrm{z}}^{2}\right)=0.120 \pm 0.002$ come significantly closer to our prediction (47)-(48). Further, our prediction $\alpha_{s}^{\overline{\mathrm{MS}}}\left(M_{\mathrm{z}}^{2}\right)=0.1192 \pm 0.0015$ is completely compatible with the world average $0.1184 \pm 0.0031$ as given in Ref. [63], but somewhat less compatible with the world average $0.1173 \pm 0.0020$ as given in Ref. 64.

\section{ACKNOWLEDGMENTS}

The work of G.C., C.D. and I.S. was supported by FONDECYT (Chile), Grant No. 1010094 (G.C.) and 8000017 (C.D. and I.S.). T.L. was supported by the BK21 Core Project.

\section{Appendix A. MODIFIED BOREL TRANSFORMS IN THE GENERAL RENORMALIZATION SCHEME}

For a function $f(y)$ with the (asymptotically divergent) expansion around $y=0$

$$
f(y)=1+\sum_{n=1}^{\infty} f_{n} y^{n},
$$

the modified Borel transform $\mathcal{F}_{f}(\zeta)$ was introduced by the authors of 42 via the following expansion:

$$
\mathcal{F}_{f}(z)=1+\sum_{n=1}^{\infty} f_{n} \frac{1}{\left(n+c_{1} z\right)\left(n-1+c_{1} z\right) \cdots\left(1+c_{1} z\right)} z^{n}
$$

where $c_{1}$ is the coefficient at the two-loop term of the $\beta$-function (111). The authors of [42 further showed that the following integral transformation connects $f(y)$ and $\mathcal{F}_{f}(z)$ :

$$
f(y)=\frac{1}{y}\left(1-c_{1} y\right) \int_{0}^{\infty} d z \mathrm{e}^{-z / y}\left(\frac{y}{z}\right)^{-c_{1} z} \frac{1}{\Gamma\left(1+c_{1} z\right)} \mathcal{F}(z)
$$

Further, they showed that there corresponds to each singularity $\sim(R-z)^{-\alpha-c_{1} R}$ of the ordinary Borel transform $F_{f}(z)=1+\sum f_{n} z^{n} / n$ ! a singularity $\sim(R-z)^{-\alpha}$ of the modified Borel tranform $\mathcal{F}_{f}(z)$ 


$$
\begin{aligned}
& F_{f}(z)=1+\sum_{n=1}^{\infty} \frac{f_{n}}{n !} z^{n} \sim(R-z)^{-\alpha-c_{1} R} \Rightarrow \\
& \mathcal{F}_{f}(z) \sim(R-z)^{-\alpha}\{1+\mathcal{O}[(R-z) \ln (1-z / R)]\} .
\end{aligned}
$$

On the other hand, the perturbative expansion of the massless Adler function $D\left(Q^{2}\right)$ has the canonical form (10), with $a=a\left(\mu^{2} ; c_{2}, c_{3}, \ldots\right)$ being the QCD couplant, and the coefficients $d_{k}$ having a specific RScl and RSch dependence $d_{k}=d_{k}\left(\xi^{2} ; c_{2}, \ldots, c_{k}\right)\left(\xi^{2} \equiv \mu^{2} / Q^{2}\right)$ determined by the requirement of the RScl and RSch independence of $D\left(Q^{2}\right)$ (cf. also [2], first entry)

$$
\begin{aligned}
d_{1}= & d_{1}^{(0)}+\beta_{0} \ln \xi^{2} \\
d_{2}= & d_{2}^{(0)}+\left(d_{1}^{2}-d_{1}^{(0) 2}\right)+c_{1}\left(d_{1}-d_{1}^{(0)}\right)-\left(c_{2}-c_{2}^{(0)}\right) \\
d_{3}= & d_{3}^{(0)}+3\left(d_{1} d_{2}-d_{1}^{(0)} d_{2}^{(0)}\right)-2\left(d_{1}^{3}-d_{1}^{(0) 3}\right)-\left(c_{1} / 2\right)\left(d_{1}^{2}-d_{1}^{(0) 2}\right) \\
& +\left(c_{2} d_{1}-c_{2}^{(0)} d_{1}^{(0)}\right)-(1 / 2)\left(c_{3}-c_{3}^{(0)}\right) \\
d_{4}= & d_{4}^{(0)}+4\left(d_{1} d_{3}-d_{1}^{(0)} d_{3}^{(0)}\right)-\left(c_{1} / 3\right)\left(d_{3}-d_{3}^{(0)}\right)+(5 / 3)\left(d_{2}^{2}-d_{2}^{(0) 2}\right) \\
& -(28 / 3)\left(d_{1}^{2} d_{2}-d_{1}^{(0) 2} d_{2}^{(0)}\right)+\left(2 c_{1} / 3\right)\left(d_{1} d_{2}-d_{1}^{(0)} d_{2}^{(0)}\right)+(1 / 3)\left(c_{2} d_{2}-c_{2}^{(0)} d_{2}^{(0)}\right) \\
& +(14 / 3)\left(d_{1}^{4}-d_{1}^{(0) 4}\right)-(4 / 3)\left(c_{2} d_{1}^{2}-c_{2}^{(0)} d_{1}^{(0) 2}\right)+\left(c_{3} d_{1}-c_{3}^{(0)} d_{1}^{(0)}\right)-(1 / 3)\left(c_{4}-c_{4}^{(0)}\right) .
\end{aligned}
$$

Here, the coefficients $d_{k}^{(0)}$ are at the RScl $\mu^{2}=Q^{2}\left(\xi^{2}=1\right)$ and in the $\operatorname{RSch} c_{2}^{(0)}, c_{3}^{(0)}, \ldots$ Comparing expansions (10) and (A.1), and inspecting the integral transformation (A.3), we may identify

$$
a=y ; \quad D\left(Q^{2}\right)=\frac{y f(y)}{\left(1-c_{1} y\right)},
$$

which immediately leads to the relations $f_{1}=d_{1}-c_{1}, f_{k}=d_{k}-c_{1} d_{k-1}(k \geq 2)$. If we use $b \equiv \beta_{0} z$ as the Borel variable, this allows us to write expansion (A.2) as

$$
\begin{aligned}
& \mathcal{F}_{D}\left(b ; \xi^{2} ; c_{2}, c_{3}, \ldots\right)=\left\{1+\frac{\left(d_{1}-c_{1}\right)}{\left(1+c_{1} b / \beta_{0}\right)}\left(\frac{b}{\beta_{0}}\right)\right. \\
& \left.+\sum_{n=2}^{\infty} \frac{\left(d_{n}-c_{1} d_{n-1}\right)}{\left(1+c_{1} b / \beta_{0}\right)\left(2+c_{1} b / \beta_{0}\right) \cdots\left(n+c_{1} b / \beta_{0}\right)}\left(\frac{b}{\beta_{0}}\right)^{n}\right\},
\end{aligned}
$$

and the integral transformation (A.3) as

$$
\begin{aligned}
D\left(Q^{2}\right) & =\frac{1}{\beta_{0}} \int_{0}^{\infty} d b \exp \left[-\frac{b}{\beta_{0} a}\right]\left(\frac{a \beta_{0}}{b}\right)^{-c_{1} b / \beta_{0}} \frac{1}{\Gamma\left(1+c_{1} b / \beta_{0}\right)} \mathcal{F}_{D}(b) \\
& =\frac{1}{\beta_{0}} \int_{0}^{\infty} d b \exp \left[-\frac{b}{\beta_{0}}\left(\frac{1}{a}+c_{1} \ln \left(c_{1} a\right)\right)\right]\left(\frac{c_{1} b}{\beta_{0}}\right)^{c_{1} b / \beta_{0}} \frac{1}{\Gamma\left(1+c_{1} b / \beta_{0}\right)} \mathcal{F}_{D}(b) .
\end{aligned}
$$

If the ordinary Borel transform $\widetilde{D}\left(b ; \xi^{2} ; c_{2}, \ldots\right)$ (21) has a singularity of the form $\sim(R-$ $\left.b / \beta_{0}\right)^{-\alpha-c_{1} R}$, then $F_{f}(z) \sim(R-z)^{-\alpha-c_{1} R}\left[1+\mathcal{O}\left((R-z)^{1}\right)\right]$, due to the simple relation $d F_{f} / d z=$ $d \widetilde{D} / d z-c_{1} \widetilde{D}$. Therefore, we can write the singularity relation between the ordinary Borel transform $\widetilde{D}$ and the modified Borel transform $\mathcal{F}_{D} \equiv \mathcal{F}_{f}$ in complete analogy with Eq. (A.4) 


$$
\begin{aligned}
\widetilde{D}\left(b ; \xi^{2} ; c_{2}, \ldots\right) & \sim(\kappa-b)^{-\alpha-c_{1} \kappa / \beta_{0}} \quad \Rightarrow \\
\mathcal{F}_{D}\left(b ; \xi^{2} ; c_{2}, \ldots\right) & \sim(\kappa-b)^{-\alpha}\{1+\mathcal{O}[(\kappa-b) \ln (1-b / \kappa)]\}
\end{aligned}
$$

where $\kappa \equiv \beta_{0} R$. We may use the subtracted Stevenson equation [2] [cf. Eq. (38)] to reexpress (partly) the expression $\left(1 / a+c_{1} \ln \left(c_{1} a\right)\right)$ in the exponential of Eq. (A.12) in terms of $\ln \left(\mu^{2} / Q^{2}\right) \equiv \ln \left(\xi^{2}\right)$ and of the invariant $\rho_{1}\left(Q^{2}\right)$ (24)

$$
\frac{1}{a}+c_{1} \ln \left(c_{1} a\right)=\rho_{1}\left(Q^{2}\right)+d_{1}^{(0)}+\beta_{0} \ln \xi^{2}-\int_{0}^{a}\left[\frac{\left(1-c_{1} x\right)}{x^{2}}+\frac{\beta_{0}}{\beta(x)}\right],
$$

where $\beta(x)$ is in the RSch considered, i.e., its expansion around $x=0$ is: $\beta\left(x ; c_{2}, \ldots\right) / \beta_{0}=$ $-x^{2}\left(1+c_{1} x+c_{2} x^{2}+\cdots\right)$. Therefore, Eq. (A.12) can be rewritten

$$
\begin{aligned}
D\left(Q^{2}\right) \equiv & D\left(a\left(\xi^{2} Q^{2} ; c_{2}, \ldots\right) ; \xi^{2} ; c_{2}, \ldots\right)=\frac{1}{\beta_{0}} \int_{0}^{\infty} d b \exp \left[-\frac{\left(\rho_{1}\left(Q^{2}\right)+\beta_{0} \ln \xi^{2}\right) b}{\beta_{0}}\right] \\
& \times \exp \left[\frac{b}{\beta_{0}} \int_{0}^{a} d x\left(\frac{\left(1-c_{1} x\right)}{x^{2}}+\frac{\beta_{0}}{\beta\left(x ; c_{2}, \ldots\right)}\right)\right] \\
& \times \frac{\left(c_{1} b / \beta_{0}\right)^{c_{1} b / \beta_{0}}}{\Gamma\left(1+c_{1} b / \beta_{0}\right)} \exp \left(-\frac{d_{1}^{(0)} b}{\beta_{0}}\right) \mathcal{F}_{D}\left(b ; \xi^{2} ; c_{2}, \ldots\right) .
\end{aligned}
$$

In the general RSch $\left(c_{2}, c_{3}, \ldots\right)$ and at the general $\mathrm{RScl} \xi^{2} \equiv \mu^{2} / Q^{2}$, the modifed Borel transform function $\mathcal{F}_{D}\left(b ; \xi^{2} ; c_{2}, \ldots\right)$ is related to the function $\mathcal{F}_{D}\left(b ; 1 ; c_{2}^{(0)}, \ldots\right)$ in the following way:

$$
\begin{aligned}
& \mathrm{e}^{-b \ln \xi^{2}} \mathcal{F}_{D}\left(b ; \xi^{2} ; c_{2}, c_{3}, \ldots\right)=\mathcal{F}_{D}\left(b ; 1 ; c_{2}^{(0)}, c_{3}^{(0)}, \ldots\right)-\frac{1}{2}\left(c_{2}-c_{2}^{(0)}\right)\left(\frac{b}{\beta_{0}}\right)^{2} \\
& +\frac{1}{12}\left[2\left(c_{2}-c_{1}^{2}\right)\left(\beta_{0} \ln \xi^{2}\right)+\left(c_{2}-c_{2}^{(0)}\right)\left(11 c_{1}-4 d_{1}^{(0)}\right)-\left(c_{3}-c_{3}^{(0)}\right)\right]\left(\frac{b}{\beta_{0}}\right)^{3} \\
& +\frac{1}{72}\left[3\left(c_{2}-c_{1}^{2}\right)\left(\beta_{0} \ln \xi^{2}\right)\left(2 d_{1}^{(0)}-\beta_{0} \ln \xi^{2}\right)+\left(22 c_{1}^{3}+3 c_{3}-25 c_{1} c_{2}\right)\left(\beta_{0} \ln \xi^{2}\right)\right. \\
& \left.-\left(c_{2}-c_{2}^{(0)}\right)\left(85 c_{1}^{2}-4 c_{2}+5 c_{2}^{(0)}-50 c_{1} d_{1}^{(0)}+9 d_{2}^{(0)}\right)+\left(c_{3}-c_{3}^{(0)}\right)\left(13 c_{1}-3 d_{1}^{(0)}\right)-\left(c_{4}-c_{4}^{(0)}\right)\right]\left(\frac{b}{\beta_{0}}\right)^{4} \\
& +\mathcal{O}\left(b^{5}\right) .
\end{aligned}
$$

This can be shown, for example, by using transformation formulas (A.5)-(A.8) in the expansion (A.10). The above formulas (A.16) and (A.15) show that the modified 't Hooft ( $\mathrm{mtH}$ ) scheme $\left(c_{k}=c_{1}^{k}, k=2,3, \ldots\right)$ is a remarkable RSch choice in the discussed Borel transforms

$$
\begin{aligned}
\mathrm{e}^{-b \ln \xi^{2}} \mathcal{F}_{D}\left(b ; \xi^{2} ; c_{1}^{2}, c_{1}^{3}, \ldots\right) & =\mathcal{F}_{D}\left(b ; 1 ; c_{1}^{2}, c_{1}^{3}, \ldots\right), \\
\frac{\left(1-c_{1} x\right)}{x^{2}}+\frac{\beta_{0}}{\beta\left(x ; c_{1}^{2}, c_{1}^{3}, \ldots\right)} & =0,
\end{aligned}
$$

and thus Eq. (A.15) reduces in this case to 


$$
\begin{aligned}
D\left(Q^{2}\right) \equiv & D\left(a\left(\xi^{2} Q^{2} ; c_{1}^{2}, c_{1}^{3} \ldots\right) ; \xi^{2} ; c_{1}^{2}, c_{1}^{3}, \ldots\right)=\frac{1}{\beta_{0}} \int_{0}^{\infty} d b \exp \left[-\frac{\left(\rho_{1}\left(Q^{2}\right)+\beta_{0} \ln \xi^{2}\right) b}{\beta_{0}}\right] \\
& \times \frac{\left(c_{1} b / \beta_{0}\right)^{c_{1} b / \beta_{0}}}{\Gamma\left(1+c_{1} b / \beta_{0}\right)} \exp \left(\frac{\left(\beta_{0} \ln \xi^{2}-d_{1}^{(0)}\right) b}{\beta_{0}}\right) \mathcal{F}_{D}\left(b ; 1 ; c_{1}^{2}, c_{1}^{3}, \ldots\right) .
\end{aligned}
$$

This is just the integral transformation (23), with expansion (25) and the constant $\tilde{c}=$ $\beta_{0} \ln \xi^{2}\left[\right.$ note that $\left.\tilde{d}_{1}=d_{1}^{(0)}=d_{1}\left(\xi^{2}=1\right)\right]$

$$
\bar{D}(b ; \tilde{c})=\mathrm{e}^{\tilde{c} b / \beta_{0}} \bar{D}(b ; 0)=\left.\frac{\left(c_{1} b / \beta_{0}\right)^{c_{1} b / \beta_{0}}}{\Gamma\left(1+c_{1} b / \beta_{0}\right)} \exp \left(\frac{\left(\tilde{c}-d_{1}^{(0)}\right) b}{\beta_{0}}\right) \mathcal{F}_{D}\left(b ; 1 ; c_{1}^{2}, c_{1}^{3}, \ldots\right)\right|_{\tilde{c}=\beta_{0} \ln \xi^{2}} .
$$

The above expression (A.19) shows the remarkable property of the mtH RSch: the whole integrand in the modified Borel transformation in the mtH RSch is RScl-independent $\left(\xi^{2}\right.$ independent). This appears the main reason why Grunberg 41] called this Borel transformation "renormalization scheme invariant." Strictly speaking, we see that this transformation may be called "renormalization scale ( $\mathrm{RScl}$ ) invariant," with the choice of the mtH renormalization scheme (RSch). On the other hand, if we abandon the mtH RSch in the general class of Borel transformations (A.15), the integrand becomes explicitly RScl-dependent

$$
\begin{aligned}
& \frac{\partial\left[\operatorname{integrand}\left(\mathrm{b} ; \xi^{2} ; c_{2}, \ldots\right)\right]}{\beta_{0} \partial \ln \xi^{2}} \\
& \propto\left(c_{1} b / \beta_{0}\right)^{c_{1} b / \beta_{0}}\left\{\left(\frac{b}{\beta_{0}}\right)\left[1+\frac{\left(1-c_{1} a\right) \beta\left(a ; c_{2}, \ldots\right)}{\beta_{0} a^{2}}\right]+\mathcal{O}\left(b^{2}\right)\right\} \\
& =\left(c_{1} b / \beta_{0}\right)^{c_{1} b / \beta_{0}}\left\{\left(\frac{b}{\beta_{0}}\right)\left[1-\left(1-c_{1} a\right)\left(1+c_{1} a+c_{2} a^{2}+\cdots\right)\right]+\mathcal{O}\left(b^{2}\right)\right\} .
\end{aligned}
$$

In order to obtain the expression for $r_{\tau}$ in terms of the modified Borel transform $\mathcal{F}_{D}\left(b ; \xi^{2} ; c_{2}, \ldots\right)$ in the general RSch, the contour integration (16) in the complex momentum plane has to be performed on the massless Adler function expression (A.15). Further, we can perform in addition the conformal transformation $b=b(w)$ of the types (41) and the ray integration trick in the $w$-plane as explained in Figs. 1,2. The procedure is analogous to the procedure leading to formula (42) in the mtH RSch, and we end up with the following formula in the general RSch:

$$
\begin{aligned}
r_{\tau}= & \frac{1}{2 \pi \beta_{0}} \operatorname{Re}\left\{e^{-\mathrm{i} \phi} \int_{0}^{1} d x \frac{d b(w)}{d w} \exp \left[-\frac{\left(\rho_{1}\left(m_{\tau}^{2} ; a_{0}\right)+\tilde{c}\right) b(w)}{\beta_{0}}\right]\right. \\
& \times \frac{\left(c_{1} b(w) / \beta_{0}\right)^{c_{1} b(w) / \beta_{0}} e^{-c_{1} b(w) / \beta_{0}}}{\Gamma\left(1+c_{1} b(w) / \beta_{0}\right)} f_{j}(b(w)) \bar{R}_{j}\left(b(w) ; \tilde{c} ; \xi^{2} ; c_{2}, \ldots\right) \\
& \times \frac{1}{(1-b(w) / 2)} \int_{-\pi}^{\pi} d y\left(1+e^{\mathrm{i} y}\right)^{3}\left(1-e^{\mathrm{i} y}\right) e^{-\mathrm{i} b(w) y} \\
& \left.\times \exp \left[\frac{b(w)}{\beta_{0}} \int_{0}^{a\left(\xi^{2} m_{\tau}^{2} \exp (\mathrm{i} y) ; c_{2}, \ldots\right)} d x\left(\frac{\left(1-c_{1} x\right)}{x^{2}}+\frac{\beta_{0}}{\beta\left(x ; c_{2}, \ldots\right)}\right)\right]\right\}\left.\right|_{w=x e^{\mathrm{i} \phi}},
\end{aligned}
$$

where the weight functions $f_{j}$ are given in Eq. (37) and the Borel transform functions $\bar{R}_{j}$ are defined in analogy with Eqs. (27)-(30) 


$$
\begin{aligned}
& \bar{R}_{1}\left(b ; \tilde{c} ; \xi^{2} ; c_{2}, \ldots\right)=(1-b / 2) \exp \left[\left(\tilde{c}+c_{1}-d_{1}^{(0)}\right) b / \beta_{0}\right] \mathrm{e}^{-b \ln \xi^{2}} \mathcal{F}_{D}\left(b ; \xi^{2} ; c_{2}, c_{3}, \ldots\right) \\
& \bar{R}_{2}\left(b ; \tilde{c} ; \xi^{2} ; c_{2}, \ldots\right)=\bar{R}_{1}\left(b ; \tilde{c} ; \xi^{2} ; c_{2}, \ldots\right) \frac{\left(1+c_{1} b / \beta_{0}\right)}{\left(2+c_{1} b / \beta_{0}\right)} \\
& \bar{R}_{3}\left(b ; \tilde{c} ; \xi^{2} ; c_{2}, \ldots\right)=\bar{R}_{1}\left(b ; \tilde{c} ; \xi^{2} ; c_{2}, \ldots\right) \frac{\left(1+c_{1} b / \beta_{0}\right)\left(2+c_{1} b / \beta_{0}\right)}{\left(3+c_{1} b / \beta_{0}\right)^{2}}
\end{aligned}
$$

We introduced an additional freedom factor $\exp \left(-\tilde{c} b / \beta_{0}\right)$ in the exponential in Eq. (A.22), which is then offset by the factor $\exp \left(+\tilde{c} b / \beta_{0}\right)$ in the functions $\bar{R}_{j}$, in analogy with the case of the mtH RSch Eq. (42). The functions $\bar{R}_{j}\left(b(w) ; \xi^{2} ; \tilde{c} ; c_{2}, \ldots\right)$ can be resummed, either as PA $[2 / 1]_{\bar{R}_{j}}(w)$ or as simple $\mathrm{N}^{3} \mathrm{LO}$ TPS. The function $\exp \left(-b \ln \xi^{2}\right) \mathcal{F}_{D}\left(b ; \xi^{2} ; c_{2}, c_{3}, \ldots\right)$, which is the source of the RScl-dependence in $\bar{R}_{j}$ 's, has only a weak RScl-dependence, as can be seen from Eq. (A.16)

$$
\begin{aligned}
e^{-b \ln \xi^{2}} \mathcal{F}_{D}\left(b ; \xi^{2} ; c_{2}, c_{3}, \ldots\right)= & \mathcal{F}_{D}\left(b ; 1 ; c_{2}, c_{3}, \ldots\right)+ \\
& \frac{1}{6}\left(c_{2}-c_{1}^{2}\right)\left(\frac{b}{\beta_{0}}\right)^{3}\left(\beta_{0} \ln \xi^{2}\right)+\mathcal{O}\left(b^{4}\right) .
\end{aligned}
$$

We further see from Eq. (A.22) that in the general RSch we cannot perform the contour integration over $d y$ analytically, in contrast to the mtH RSch Eq. (42). 


\section{REFERENCES}

[1] S. J. Brodsky, G. P. Lepage and P. B. Mackenzie, Phys. Rev. D 28, 228 (1983).

[2] P. M. Stevenson, Phys. Rev. D 23, 2916 (1981); Phys. Lett. 100B, 61 (1981); Nucl. Phys. B203, 472 (1982).

[3] G. Grunberg, Phys. Lett. 95B, 70 (1980), 110B, 501(E) (1982); 114B, 271 (1982); Phys. Rev. D 29, 2315 (1984).

[4] A. L. Kataev, N. V. Krasnikov and A. A. Pivovarov, Nucl. Phys. B 198, 508 (1982) [Erratum-ibid. B 490, 505 (1982)] [hep-ph/9612326].

[5] A. Dhar and V. Gupta, Phys. Rev. D 29, 2822 (1984); V. Gupta, D. V. Shirkov, and O. V. Tarasov, Int. J. Mod. Phys. A 6, 3381 (1991).

[6] H. Lu and S. J. Brodsky, Phys. Rev. D 48, 3310 (1993) hep-ph/9211254; S. J. Brodsky and H. J. Lu, ibid. 51, 3652 (1995) hep-ph/9405218; S. J. Brodsky, G. T. Gabadadze, A. L. Kataev and H. J. Lu, Phys. Lett. B 372, 133 (1996) hep-ph/9512367; S. Groote, J. G. Körner, A. A. Pivovarov and K. Schilcher, Phys. Rev. Lett. 79, 2763 (1997) [hepph/9703208]; S. J. Brodsky, J. R. Peláez and N. Toumbas, Phys. Rev. D 60, 037501 (1999) hep-ph/9810424. M. Binger, C. Ji, and D. G. Robertson, ibid. 61, 114011 (2000) hep-ph/9912241.

[7] C. J. Maxwell, hep-ph/9908463; C. J. Maxwell and A. Mirjalili, Nucl. Phys. B577, 209 (2000) hep-ph/0002204.

[8] C. J. Maxwell and D. G. Tonge, Nucl. Phys. B 535, 19 (1998) hep-ph/9705314.

[9] C. J. Maxwell and A. Mirjalili, hep-ph/0103164.

[10] J. G. Körner, F. Krajewski, and A. A. Pivovarov, Phys. Rev. D 63, 036001 (2001) hep-ph/0002166.

[11] D. V. Shirkov and I. L. Solovtsov, Phys. Rev. Lett. 79, 1209 (1997) hep-ph/9704333; K. A. Milton, I. L. Solovtsov, and O. P. Solovtsova, Phys. Lett. B 439, 421 (1998) hep-ph/9809510; Phys. Rev. D 64, 016005 (2001) hep-ph/0102254; Eur. Phys. J. C 14, 495 (2000) [hep-ph/0003030]; I. L. Solovtsov and D. V. Shirkov, Phys. Lett. B 442, 344 (1998) hep-ph/9711251; Theor. Math. Phys. 120, 1220 (1999) hep-ph/9909305;

D. V. Shirkov, ibid. 127, 409 (2001) hep-ph/0012283.

[12] D. S. Kourashev, hep-ph/9912410; hep-ph/0010072.

[13] S. J. Brodsky, E. Gardi, G. Grunberg, and J. Rathsman, Phys. Rev. D 63, 094017 (2001) hep-ph/0002065.

[14] I. L. Solovtsov, Phys. Lett. B 327, 335 (1994); 340, 245 (1994); K. A. Milton, I. L. Solovtsov, and O. P. Solovtsova, Eur. Phys. J. C 13, 497 (2000) [hep-ph/0003031.

[15] T. Lee, Phys. Rev. D 56, 1091 (1997) hep-th/9611010; Phys. Lett. B 462, 1 (1999) hep-ph/9908225.

[16] I. Caprini and J. Fischer, Phys. Rev. D 60, 054014 (1999) hep-ph/9811367.

[17] M. A. Samuel, J. Ellis, and M. Karliner, Phys. Rev. Lett. 74, 4380 (1995) hepph/9503411]; J. Ellis, E. Gardi, M. Karliner, and M. A. Samuel, Phys. Lett. B 366, 268 (1996) hep-ph/9509312; Phys. Rev. D 54, 6986 (1996) hep-ph/9607404.

[18] E. Gardi, Phys. Rev. D 56, 68 (1997) hep-ph/9611453]; S. J. Brodsky, J. Ellis, E. Gardi, M. Karliner, and M. A. Samuel, ibid. 56, 6980 (1997) hep-ph/9706467.

[19] T. G. Steele and V. Elias, Mod. Phys. Lett. A 13, 3151 (1998) hep-ph/9902217.

[20] G. Cvetič, Nucl. Phys. B 517, 506 (1998) hep-ph/9711406; Phys. Rev. D 57, R3209 
(1998) [hep-ph/9711487; G. Cvetič and R. Kögerler, Nucl. Phys. B 522, 396 (1998) thepph/9802248]; G. Cvetič, Nucl. Phys. B (Proc. Suppl.) 74, 333 (1999) hep-ph/9808273].

[21] G. Cvetič and R. Kögerler, Phys. Rev. D 63, 056013 (2001) [hep-ph/0006098]; G. Cvetič, Phys. Lett. B 486, 100 (2000) hep-ph/0003123.

[22] G. Cvetič and T. Lee, Phys. Rev. D 64, 014030 (2001) [hep-ph/0101297.

[23] M. A. Shifman, A. I. Vainshtein and V. I. Zakharov, Nucl. Phys. B 147, 385 (1979); ibid 448 (1979); ibid 519 (1979).

[24] E. Braaten, S. Narison, and A. Pich, Nucl. Phys. B 373, 581 (1992).

[25] Y. L. Dokshitzer, G. Marchesini, and B. R. Webber, Nucl. Phys. B 469, 93 (1996) thepph/9512336; E. Gardi and G. Grunberg, JHEP 9911, 016 (1999) hep-ph/9908458; G. Grunberg, ibid. 9811, 006 (1998) hep-ph/9807494.

[26] T. Lee, hep-ph/0104306.

[27] R. Barate et al. [ALEPH Collaboration], Eur. Phys. J. C 4, 409 (1998).

[28] R. Barate et al. [ALEPH Collaboration], Eur. Phys. J. C 11, 599 (1999) hepex/9903015; ibid. 10, 1 (1999) [hep-ex/9903014.

[29] M. Davier, hep-ex/9912044.

[30] G. Abbiendi et al. [OPAL Collaboration], Eur. Phys. J. C 13, 197 (2000) hepex/9908013.

[31] S. J. Richichi et al. [CLEO Collaboration], Phys. Rev. D 60, 112002 (1999) hepex/9810026.

[32] M. Davier, private communication; and talk given at the Sixth International Workshop on Tau Lepton Physics (Tau'2000 Workshop), September 2000, Victoria, Canada (to appear on http://tau2000.phys.uvic.ca/).

[33] D. E. Groom et al. [Particle Data Group Collaboration], Eur. Phys. J. C 15, 1 (2000).

[34] C. S. Lam and T. M. Yan, Phys. Rev. D 16, 703 (1977).

[35] K. Schilcher and M. D. Tran, Phys. Rev. D 29, 570 (1984).

[36] S. Narison and A. Pich, Phys. Lett. B 211, 183 (1988).

[37] E. Braaten, Phys. Rev. Lett. 60, 1606 (1988); Phys. Rev. D 39, 1458 (1989).

[38] A. Pich, Hadronic tau-decays and QCD, in Proc. Workshop on Tau Lepton Physics, Orsay, France, September 1990, eds. M. Davier and B. Jean-Marie (Ed. Frontières, Paris, 1991), p.321 (see also preprint CERN-TH-5940-90, KEK Library).

[39] A. A. Pivovarov, Z. Phys. C 53, 461 (1992) [Yad. Fiz. 54, 1114 (1991), Sov. J. Nucl. Phys. 54, 676 (1992)]; F. Le Diberder and A. Pich, Phys. Lett. B 286, 147 (1992).

[40] A. Pich and J. Prades, JHEP9806, 013 (1998) [hep-ph/9804462]; ibid. 9910, 004 (1999) hep-ph/9909244.

[41] G. Grunberg, Phys. Lett. B 304, 183 (1993).

[42] L. S. Brown and L. G. Yaffe, Phys. Rev. D 45, 398 (1992); L. S. Brown, L. G. Yaffe and C. Zhai, ibid. 46, 4712 (1992) hep-ph/9205213].

[43] E. Braaten and C. Li, Phys. Rev. D 42, 3888 (1990).

[44] W. J. Marciano and A. Sirlin, Phys. Rev. Lett. 61, 1815 (1988).

[45] K. G. Chetyrkin, S. Narison, and V. I. Zakharov, Nucl. Phys. B 550, 353 (1999) thep$\mathrm{ph} / 9811275$.

[46] S. J. Huber, M. Reuter, and M. G. Schmidt, Phys. Lett. B 462, 158 (1999) hepph/9906358].

[47] C. A. Dominguez and K. Schilcher, Phys. Rev. D 61, 114020 (2000) hep-ph/9903483. 
[48] K. Maltman, Phys. Lett. B 440, 367 (1998) [hep-ph/9901239.

[49] C. A. Dominguez and K. Schilcher, Phys. Lett. B 448, 93 (1999) hep-ph/9811261.

[50] K. G. Chetyrkin, A. L. Kataev, and F. V. Tkachev, Phys. Lett. B 85, 277 (1979); M. Dine and J. Sapirstein, Phys. Rev. Lett. 43, 668 (1979); W. Celmaster and R. J. Gonsalves, ibid. 44, 560 (1980).

[51] S. G. Gorishny, A. L. Kataev and S. A. Larin, Phys. Lett. B 259, 144 (1991); L. R. Surguladze and M. A. Samuel, Phys. Rev. Lett. 66, 560 (1991) [Erratum-ibid. 66, 2416 (1991)].

[52] M. Beneke and V. M. Braun, Phys. Lett. B 348, 513 (1995) [hep-ph/9411229]; P. Ball, M. Beneke, and V. M. Braun, Nucl. Phys. B 452, 563 (1995) hep-ph/9502300.

[53] M. Neubert, Nucl. Phys. B 463, 511 (1996) hep-ph/9509432.

[54] C. N. Lovett-Turner and C. J. Maxwell, Nucl. Phys. B 452, 188 (1995) hep$\mathrm{ph} / 9505224$.

[55] A. H. Mueller, Nucl. Phys. B 250, 327 (1985).

[56] T. van Ritbergen, J. A. Vermaseren, and S. A. Larin, Phys. Lett. B 400, 379 (1997) hep-ph/9701390.

[57] G. Altarelli, P. Nason, and G. Ridolfi, Z. Phys. C 68, 257 (1995) hep-ph/9501240.

[58] George A. Baker, Jr. and Peter Graves-Morris, Padé Approximants, 2nd edition, (Encyclopedia of Mathematics and Its Applications, Vol. 59), edited by Gian-Carlo Rota (Cambridge University Press, 1996).

[59] U. D. Jentschura and G. Soff, J. Phys. AA 34, 1451 (2001) hep-ph/0006089.

[60] K. G. Chetyrkin, B. A. Kniehl, and M. Steinhauser, Phys. Rev. Lett. 79, 2184 (1997) hep-ph/9706430.

[61] S. Groote, J. G. Körner, and A. A. Pivovarov, Mod. Phys. Lett. A 13, 637 (1998) hep-ph/9703268.

[62] B. V. Geshkenbein, B. L. Ioffe, and K. N. Zyablyuk, Phys. Rev. D (to be published) hep-ph/0104048.

[63] S. Bethke, J. Phys. G G26, R27 (2000) hep-ex/0004021.

[64] I. Hinchliffe and A. V. Manohar, Ann. Rev. Nucl. Part. Sci. 50, 643 (2000) hep$\mathrm{ph} / 0004186$. 


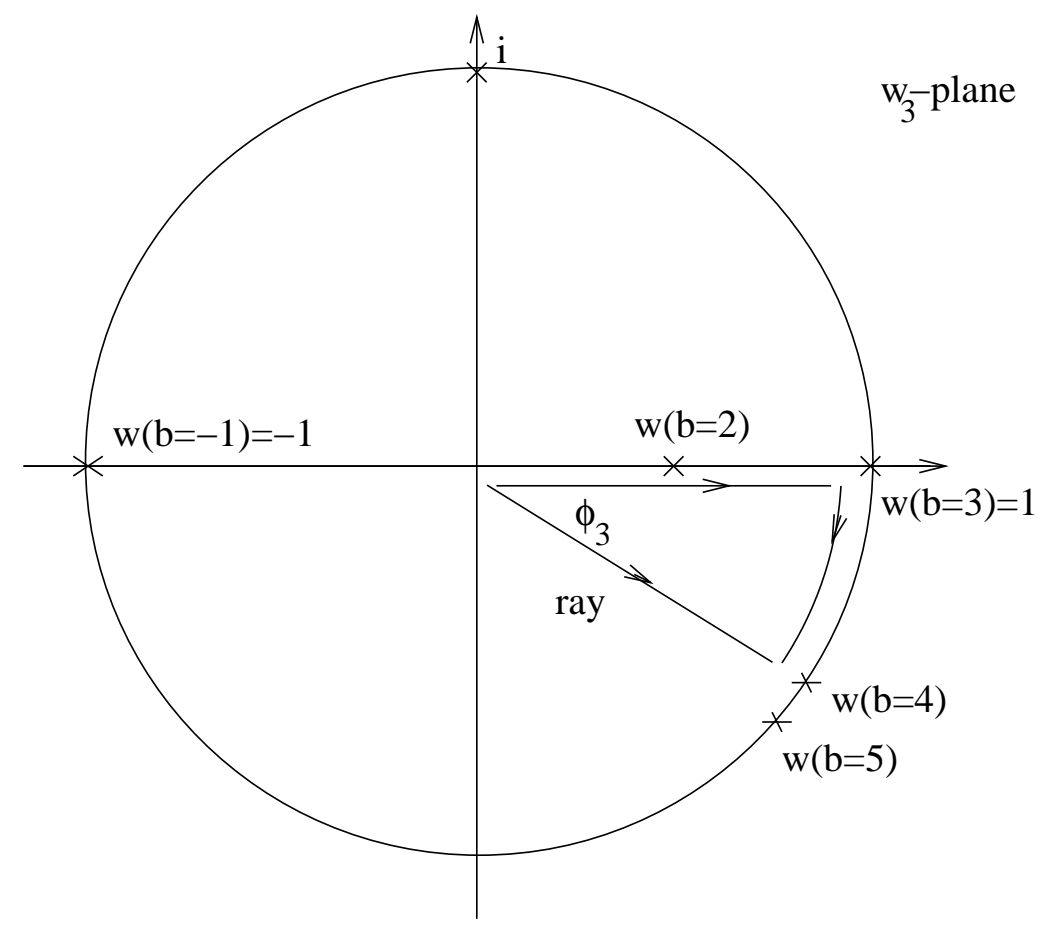

FIG. 1. Integration in the $w_{3}$-plane along the ray $w_{3}=x \exp \left(-\mathrm{i} \phi_{3}\right)\left(0<x<1, \phi_{3}=0.50536\right)$ gives the same result as the integration parallel to the positive real axis $(0<w<1)$ and arc $w=\exp \left(-\mathrm{i} \phi^{\prime}\right)$ $\left(0<\phi^{\prime}<\phi_{3}\right)$.

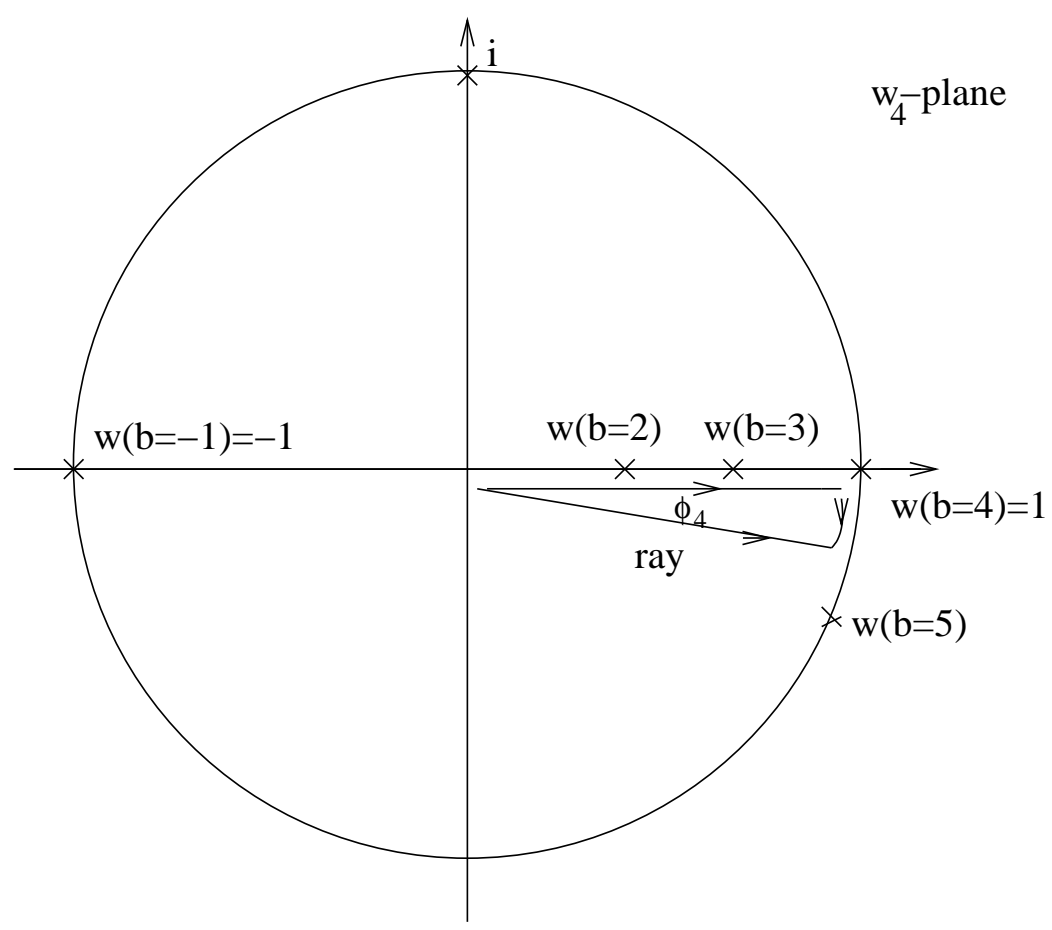

FIG. 2. Integration in the $w_{4}-$ plane along the ray $w_{4}=x \exp \left(-\mathrm{i} \phi_{4}\right)\left(0<x<1, \phi_{4}=0.1\right)$. 


\begin{tabular}{cccc}
\hline \hline$(j, k) ;$ approximant & $r_{\tau}\left[\alpha_{s}\left(m_{\tau}^{2}\right)=0.325 ; 0.326\right]$ & $\tilde{c}$ & comments \\
\hline \hline$(1,3) ;[2 / 1]$ & $0.19543 ; 0.19641$ & +0.28 & $w^{\text {pole }} \approx-1$. \\
\hline$(1,4) ;[2 / 1]$ & $0.19524 ; 0.19621$ & +0.17 & $w^{\text {pole } \approx-1 .}$ \\
\hline$(2,3) ;[2 / 1]$ & $0.19533 ; 0.19631$ & -0.27 & $w^{\text {pole } \approx-1 .}$ \\
\hline$(2,4) ;[2 / 1]$ & $0.19531 ; 0.19629$ & -0.28 & $w^{\text {pole } \approx-1 .}$ \\
\hline$(3,3) ;[2 / 1]$ & $0.19570 ; 0.19668$ & -0.15 & $w^{\text {pole } \approx-1 .}$ \\
\hline$(3,4) ;[2 / 1]$ & $0.19554 ; 0.19651$ & -0.22 & $w^{\text {pole } \approx-1 .}$ \\
\hline \hline$(2,3) ;[2 / 1]$ & $0.19470 ; 0.19567$ & -0.71 & $w^{\text {pole } \approx-1 .}$ \\
\hline$(2,4) ;[2 / 1]$ & $0.19466 ; 0.19563$ & -0.89 & $w^{\text {pole } \approx-1 .}$ \\
\hline \hline$(1,3) ;[2 / 1]$ & $0.19503 ; 0.19600$ & +1.95 & $w^{\text {pole }} \approx+1$. \\
\hline$(1,4) ;[2 / 1]$ & $0.19587 ; 0.19686$ & $w^{\text {pole }} \approx+0.6$ \\
\hline$(2,3) ;[2 / 1]$ & $0.19648 ; 0.19748$ & $w^{\text {pole }} \approx+1$. \\
\hline$(2,4) ;[2 / 1]$ & $0.19746 ; 0.19847$ & +2.275 & $w^{\text {pole }} \approx+0.6$ \\
\hline$(3,3) ;[2 / 1]$ & $0.19686 ; 0.19786$ & +1.23 & $w^{\text {pole }} \approx+1$. \\
\hline$(3,4) ;[2 / 1]$ & $0.19792 ; 0.19895$ & +1.61 & $w^{\text {pole }} \approx+0.6$ \\
\hline \hline$(1,3) ;$ TPS & $0.19449 ; 0.19546$ & +0.97 & local max. \\
\hline$(1,4) ;$ TPS & $0.19433 ; 0.19529$ & +1.34 & local max. \\
\hline$(2,3) ;$ TPS & $0.19545 ; 0.19643$ & +0.92 & local max. \\
\hline$(2,4) ;$ TPS & $0.19516 ; 0.19614$ & +0.54 & local max. \\
\hline$(3,3) ;$ TPS & $0.19568 ; 0.19666$ & +0.47 & local max. \\
\hline$(3,4) ;$ TPS & $0.19535 ; 0.19633$ & +0.12 & local max. \\
\hline \hline arithm. average $\bar{r}_{\tau}$ & $0.19560 ; 0.19658$ & -0.01 & \\
\hline \hline
\end{tabular}

TABLE I. The results $r_{\tau}$ of calculations according to Eq. (42), using $d_{3}^{(0)}=25$, employing the conformal transformations (41) $(k=3,4)$ and the resummations of $\bar{R}_{j}\left(w_{k}\right)$ (27)-(30) $(j=1,2,3)$ as PA [2/1] and as $\left(\mathrm{N}^{3} \mathrm{LO}\right)$ TPS. The constant $\tilde{c}$ was varied so as to achieve either $w_{k}^{\text {pole }}=-1$ or $w_{3}^{\text {pole }}=+1\left(w_{4}^{\text {pole }}=+0.6\right)$ when using PA's [2/1], or stationarity according to the PMS condition 443) when using $\left(\mathrm{N}^{3} \mathrm{LO}\right)$ TPS. The results $r_{\tau}$ are given for the choices $\alpha_{s}\left(m_{\tau}^{2} ; \overline{\mathrm{MS}}\right)=0.325$ and 0.326 . The central measured value $r_{\tau}=0.1960$ (18) is achieved by the arithmetic average $\bar{r}_{\tau}$ at $\alpha_{s}\left(m_{\tau}^{2} ; \overline{\mathrm{MS}}\right)=0.3254$. 


\begin{tabular}{cccc}
\hline \hline$(j, k) ;$ approximant & $r_{\tau}\left[\alpha_{s}\left(m_{\tau}^{2}\right)=0.331 ; 0.332\right]$ & $\tilde{c}$ & comments \\
\hline \hline$(1,3) ;[2 / 1]$ & $0.19625 ; 0.19717$ & +1.20 & $w^{\text {pole }} \approx-1$. \\
\hline$(1,4) ;[2 / 1]$ & $0.19600 ; 0.19692$ & +1.10 & $w^{\text {pole } \approx-1 .}$ \\
\hline$(2,3) ;[2 / 1]$ & $0.19712 ; 0.19806$ & +0.83 & $w^{\text {pole } \approx-1 .}$ \\
\hline$(2,4) ;[2 / 1]$ & $0.19688 ; 0.19781$ & +0.74 & $w^{\text {pole } \approx-1 .}$ \\
\hline$(3,3) ;[2 / 1]$ & $0.19776 ; 0.19871$ & +0.55 & $w^{\text {pole } \approx-1 .}$ \\
\hline$(3,4) ;[2 / 1]$ & $0.19750 ; 0.19844$ & +0.46 & $w^{\text {pole } \approx-1 .}$ \\
\hline \hline$(1,3) ;[2 / 1]$ & $0.19601 ; 0.19693$ & +2.52 & $w^{\text {pole } \approx+1 .}$ \\
\hline$(1,4) ;[2 / 1]$ & $0.19687 ; 0.19781$ & +2.83 & $w^{\text {pole }} \approx+0.6$ \\
\hline$(2,3) ;[2 / 1]$ & $0.19758 ; 0.19853$ & +1.98 & $w^{\text {pole }} \approx+1$. \\
\hline$(2,4) ;[2 / 1]$ & $0.19856 ; 0.19953$ & +2.30 & $w^{\text {pole }} \approx+0.6$ \\
\hline$(3,3) ;[2 / 1]$ & $0.19831 ; 0.19927$ & +1.64 & $w^{\text {pole }} \approx+1$. \\
\hline$(3,4) ;[2 / 1]$ & $0.19942 ; 0.20040$ & +1.97 & $w^{\text {pole }} \approx+0.6$ \\
\hline \hline$(1,3) ;$ TPS & $0.19543 ; 0.19634$ & +1.6 & local max. \\
\hline$(1,4) ;$ TPS & $0.19525 ; 0.19616$ & +1.3 & local max. \\
\hline$(2,3) ;$ TPS & $0.19666 ; 0.19759$ & +1.2 & local max. \\
\hline$(2,4) ;$ TPS & $0.19640 ; 0.19733$ & +0.9 & local max. \\
\hline$(3,3) ;$ TPS & $0.19732 ; 0.19826$ & +0.9 & local max. \\
\hline$(3,4) ;$ TPS & $0.19703 ; 0.19797$ & +0.6 & local max. \\
\hline \hline arithm. average $\bar{r}_{\tau}$ & $0.19702 ; 0.19796$ & & \\
\hline \hline
\end{tabular}

TABLE II. Results analogous to those of Table $\mathbb{\|}$, but for the case $d_{3}^{(0)}=15$. The results $r_{\tau}$ are given for the choices $\alpha_{s}\left(m_{\tau}^{2} ; \overline{\mathrm{MS}}\right)=0.331$ and 0.332 . The central measured value $r_{\tau}=0.1960$ (18) is achieved by the arithmetic average $\bar{r}_{\tau}$ at $\alpha_{s}\left(m_{\tau}^{2} ; \overline{\mathrm{MS}}\right)=0.3299$. 


\begin{tabular}{cccc}
\hline \hline$(j, k) ;$ approximant & $r_{\tau}\left[\alpha_{s}\left(m_{\tau}^{2}\right)=0.331 ; 0.332\right]$ & $\tilde{c}$ & comments \\
\hline \hline$(1,3) ;[2 / 1]$ & $0.19716 ; 0.19823$ & +1.02 & $w^{\text {pole }} \approx+1$. \\
\hline$(1,4) ;[2 / 1]$ & $0.19780 ; 0.19888$ & +1.37 & $w^{\text {pole } \approx+0.6}$ \\
\hline \hline$(1,3) ;$ TPS & $0.19744 ; 0.19852$ & +0.025 & $\partial r_{\tau} / \partial \tilde{c} \approx-2.66 \cdot 10^{-3}$ \\
\hline$(1,4) ;$ TPS & $0.19645 ; 0.19751$ & +0.0 & $\partial r_{\tau} / \partial \tilde{c} \approx-2.92 \cdot 10^{-3}$ \\
\hline$(2,3) ;$ TPS & $0.19603 ; 0.19708$ & +0.375 & $\partial r_{\tau} / \partial \tilde{c} \approx-3.18 \cdot 10^{-3}$ \\
\hline$(2,4) ;$ TPS & $0.19558 ; 0.19662$ & +0.075 & $\partial r_{\tau} / \partial \tilde{c} \approx-3.09 \cdot 10^{-3}$ \\
\hline$(3,3) ;$ TPS & $0.19651 ; 0.19757$ & -0.19 & $\partial r_{\tau} / \partial \tilde{c} \approx-2.37 \cdot 10^{-3}$ \\
\hline$(3,4) ;$ TPS & $0.19602 ; 0.19707$ & -0.49 & $\partial r_{\tau} / \partial \tilde{c} \approx-2.29 \cdot 10^{-3}$ \\
\hline \hline arithm. average $\bar{r}_{\tau}$ & $0.19662 ; 0.19769$ & \\
\hline \hline
\end{tabular}

TABLE III. Results analogous to those of Table $\mathbb{1}$, but for the case $d_{3}^{(0)}=35$. The results $r_{\tau}$ are given for the choices $\alpha_{s}\left(m_{\tau}^{2} ; \overline{\mathrm{MS}}\right)=0.320$ and 0.321 . In the approach with the $\left(\mathrm{N}^{3} \mathrm{LO}\right)$ TPS of $\bar{R}_{j}\left(w_{k}\right)$, the PMS condition (43) is never exactly satisfied; there, the values of $\tilde{c}$ were chosen so that the (negative) slope $\partial r_{\tau} / \partial \tilde{c}$ is the least steep. The central measured value $r_{\tau}=0.1960(18)$ is achieved by the arithmetic average $\bar{r}_{\tau}$ at $\alpha_{s}\left(m_{\tau}^{2} ; \overline{\mathrm{MS}}\right)=0.3194$.

\begin{tabular}{cccr}
\hline \hline$(j, k) ;$ approximant & $r_{\tau}\left[\alpha_{s}\left(m_{\tau}^{2}\right)=0.329 ; 0.330\right]$ & $\tilde{c}$ & comments \\
\hline \hline$(2,4) ;[2 / 1]$ & $0.19521 ; 0.19613$ & +0.01 & $w^{\text {pole }} \approx-1$. \\
\hline \hline$(3,3) ;[2 / 1]$ & $0.19656 ; 0.19750$ & +0.707 & $w^{\text {pole }} \approx+1$. \\
\hline$(3,3) ;[2 / 1]$ & $0.19770 ; 0.19866$ & +1.25 & $w^{\text {pole } \approx+1 .}$ \\
\hline \hline$(2,3) ;$ TPS & $0.19668 ; 0.19763$ & +1.1 & local max. \\
\hline$(2,4) ;$ TPS & $0.19633 ; 0.19727$ & +0.8 & local max. \\
\hline$(3,3) ;$ TPS & $0.19672 ; 0.19767$ & +1.05 & local max. \\
\hline$(3,4) ;$ TPS & $0.19638 ; 0.19732$ & +0.75 & local max. \\
\hline \hline arithm. average $\bar{r}_{\tau}$ & $0.19651 ; 0.19745$ & & \\
\hline \hline
\end{tabular}

TABLE IV. Results analogous to those of Table [, but for the different RSch: $c_{2}=c_{1}^{2}+3.4, c_{k}=c_{1}^{k}$ $(k=3,4, \ldots)$. Expression A.22 is used to calculate $r_{\tau}$. RScl parameter is $\xi^{2} \equiv \mu^{2} / Q^{2}=1\left[Q^{2}=m_{\tau}^{2} \exp (\mathrm{i} y)\right]$; $d_{3}^{(0)}=25$. The results $r_{\tau}$ are given for the choices $\alpha_{s}\left(m_{\tau}^{2} ; \overline{\mathrm{MS}}\right)=0.329$ and 0.330 . The central measured value $r_{\tau}=0.1960(18)$ is achieved by the arithmetic average $\bar{r}_{\tau}$ at $\alpha_{s}\left(m_{\tau}^{2} ; \overline{\mathrm{MS}}\right)=0.3285$. 


\begin{tabular}{cccc}
\hline \hline$(j, k) ;$ approximant & $r_{\tau}\left[\alpha_{s}\left(m_{\tau}^{2}\right)=0.327 ; 0.328\right]$ & $\tilde{c}$ & comments \\
\hline \hline$(1,3) ;[2 / 1]$ & $0.19478 ; 0.19570$ & +1.50 & $w^{\text {pole }} \approx-1$. \\
\hline$(1,4) ;[2 / 1]$ & $0.19453 ; 0.19545$ & +1.40 & $w^{\text {pole } \approx-1 .}$ \\
\hline$(2,3) ;[2 / 1]$ & $0.19582 ; 0.19676$ & +1.11 & $w^{\text {pole } \approx-1 .}$ \\
\hline$(2,4) ;[2 / 1]$ & $0.19558 ; 0.19652$ & +1.01 & $w^{\text {pole } \approx-1 .}$ \\
\hline$(3,3) ;[2 / 1]$ & $0.19654 ; 0.19749$ & +0.80 & $w^{\text {pole } \approx-1 .}$ \\
\hline$(3,4) ;[2 / 1]$ & $0.19628 ; 0.19723$ & +0.70 & $w^{\text {pole } \approx-1 .}$ \\
\hline \hline$(1,3) ;[2 / 1]$ & $0.19456 ; 0.19549$ & +2.77 & $w^{\text {pole } \approx+1 .}$ \\
\hline$(1,4) ;[2 / 1]$ & $0.19538 ; 0.19632$ & +3.07 & $w^{\text {pole }} \approx+0.6$ \\
\hline$(2,3) ;[2 / 1]$ & $0.19612 ; 0.19708$ & +2.25 & $w^{\text {pole }} \approx+1$. \\
\hline$(2,4) ;[2 / 1]$ & $0.19708 ; 0.19805$ & +2.56 & $w^{\text {pole }} \approx+0.6$ \\
\hline$(3,3) ;[2 / 1]$ & $0.19695 ; 0.19792$ & +1.90 & $w^{\text {pole } \approx+1 .}$ \\
\hline$(3,4) ;[2 / 1]$ & $0.19796 ; 0.19894$ & +2.205 & $w^{\text {pole }} \approx+0.6$ \\
\hline \hline$(1,3) ;$ TPS & $0.19400 ; 0.19492$ & +1.9 & local max. \\
\hline$(1,4) ;$ TPS & $0.19383 ; 0.19475$ & +1.5 & local max. \\
\hline$(2,3) ;$ TPS & $0.19528 ; 0.19622$ & +1.45 & local max. \\
\hline$(2,4) ;$ TPS & $0.19505 ; 0.19598$ & +1.1 & local max. \\
\hline$(3,3) ;$ TPS & $0.19602 ; 0.19696$ & +1.1 & local max. \\
\hline$(3,4) ;$ TPS & $0.19576 ; 0.19670$ & +0.8 & \\
\hline \hline arithm. average $\bar{r}_{\tau}$ & $0.19564 ; 0.19658$ & & \\
\hline \hline
\end{tabular}

TABLE V. Results analogous to those of Table IV, but for the RSch: $c_{3}=c_{1}^{3}+31.0, c_{k}=c_{1}^{k}$ $(k=2,4,5, \ldots)$. Expression (A.22) is used to calculate $r_{\tau}$. RScl parameter is $\xi^{2}=1 ; d_{3}^{(0)}=25$. The results $r_{\tau}$ are given for the choices $\alpha_{s}\left(m_{\tau}^{2} ; \overline{\mathrm{MS}}\right)=0.327$ and 0.328 . The central measured value $r_{\tau}=0.1960$ (18) is achieved by the arithmetic average $\bar{r}_{\tau}$ at $\alpha_{s}\left(m_{\tau}^{2} ; \overline{\mathrm{MS}}\right)=0.3274$. 